\title{
Desarrollo de Un SISTEMA DE SOPORTE DE DECISIONES PARA UNA EMPRESA DE TRANSPORTE. UNA APLICACIÓN DEL REVENUE MANAGEMENT Y DE LA PROGRAMACIÓN NO LINEAL
}

\author{
José Antonio Mendoza Quispe \\ jmendoza@agosac.com \\ Asesores en Gestión de Operaciones S.A. Lima, Perú
}

\section{Resumen}

En el presente artículo de investigación se desarrollará un enfoque de planificación que permita a las empresas de transporte interprovincial terrestre de pasajeros, por medio del uso de las tecnologías de información, mantener una programación eficiente de sus viajes, gestionar la disponibilidad de sus recursos a mediano y largo plazo y aplicar el revenue management para obtener mayores ingresos económicos. El desarrollo de un sistema de soporte de decisiones en este sector permitirá que la programación de viajes se enfoque en optimizar la contribución marginal total a largo plazo, es decir, la diferencia entre los ingresos y los costos variables; así como la realización de una correcta asignación de recursos a los viajes, y que los precios establecidos para los pasajes sean los adecuados para alcanzar las metas de la empresa.

Palabras clave: sistema de soporte de decisiones / programación no lineal / revenue management

\section{Abstract}

This study develops a planning approach to enable interprovincial land-transport companies to maintain an efficient travel schedule, considering the availability of their medium and long-term resources and the application of revenue management to obtain higher incomes through the use of information technologies. The development of a decision-support system in this sector will allow travel schedule focuses for optimizing the total marginal contribution in the long term, i.e. the difference between incomes and variable costs, as well as an appropriate allocation of resources to the trips, and that the fares established are adequate for the company to reach its goals.

Keywords: decision support system / non-linear programming / revenue management 


\section{Introducción}

En la actualidad existe una gran cantidad de empresas de transporte interprovincial de pasajeros en el Perú, cuyos factores clave son la fuerza laboral de la empresa, el servicio de viajes que ofrecen y los procesos involucrados para realizar el servicio, tales como: la programación de los viajes, la fijación de precios y el manejo de recursos. Estos procesos se vuelven más laboriosos y complicados de realizar según el tamaño de la empresa y la cantidad de rutas que se atienden.

La programación de viajes en este tipo de empresas está directamente relacionada con la asignación de la tripulación y los buses. Las empresas de transporte interprovincial asignan frecuentemente dos conductores a un vehículo para cada trayecto, debido a que la mayoría de rutas tienen más de cinco horas de viaje y el decreto supremo N. ${ }^{\circ}$ 009-2004MTC indica, en el artículo 121, que un conductor no puede estar ejerciendo su labor más de cinco horas continuas durante el día, y no más de cuatro horas por la noche.

En el Perú, más del 50 \% del transporte interprovincial opera informalmente, de acuerdo al Consejo Nacional de Transporte Terrestre; la mayoría no trabaja con procesos definidos ni enfocados en las mejores prácticas. En la tabla 1, basada en la información proporcionada por el Centro de Investigación, Desarrollo y Asesoría de Transporte Terrestre (CIDDAT), se muestra a las nueve empresas del Perú que proporcionan la mayor oferta de viajes periódicamente a diciembre del 2015 y la cantidad de unidades que poseen.

Tabla 1. Ranking de empresas con mayor oferta de viajes a diciembre del 2015.

\begin{tabular}{|c|l|c|}
\hline \multicolumn{1}{|c|}{ Empresa } & Cantidad de unidades \\
\hline 1 & Flores & 461 \\
\hline 2 & Cruz Del Sur & 359 \\
\hline 3 & Perú Bus & 402 \\
\hline 4 & Línea & 486 \\
\hline 5 & ClVA S.A.C. & 218 \\
\hline 6 & Movil Tours & 175 \\
\hline 7 & Zeballos & 117 \\
\hline 8 & Cromotex S.A.C. & 112 \\
\hline 9 & Palomino & 165 \\
\hline
\end{tabular}

Fuente: Elaboración propia a partir de Romainville (2015) 
Además, en el mencionado estudio se concluyó que "el 48 \% de la oferta de buses, en el transporte internacional y el 36 \% en el transporte de trabajadores, a nivel nacional, estuvieron en manos de dichos grupos empresariales de transporte terrestre" (Romainville, 2015). Es importante mencionar que no existen muchas investigaciones en este sector.

\subsection{Objetivos de estudio}

A continuación se presentan el objetivo general y los objetivos específicos planteados para el presente estudio.

\subsubsection{Objetivo general}

Desarrollar un sistema de soporte de decisiones que permita a las empresas de transporte terrestre interprovincial realizar una programación eficiente de viajes y una correcta determinación de los precios ofertados, de tal forma que se obtenga una mayor contribución marginal total, se reduzcan los errores y sobrecostos asociados a una inadecuada programación.

\subsubsection{Objetivos específicos}

Para el logro del objetivo general planteado se formularon los siguientes objetivos específicos:

i. Realizar un diagnóstico de la empresa que permita determinar los recursos disponibles, las rutas de transporte ofertadas y los costos asociados.

ii. Formular un modelo matemático que permita obtener una programación eficiente de los viajes, con el objetivo de maximizar la contribución marginal.

iii. Realizar pruebas para validar que los resultados del modelo se ajusten a la realidad del negocio.

iv. Determinar el impacto de realizar una programación de viajes mediante el enfoque planteado.

\subsection{Alcance}

En la presente investigación se busca mejorar la planificación de las empresas en el sector de transporte con el desarrollo de un sistema de soporte de decisiones que genere una óptima programación de viajes según los recursos disponibles, las rutas establecidas y las personas asignadas. También se encargará de proponer el precio adecuado de los pasajes en relación a la demanda estimada de viajes que se tenga, para lo cual se establecen los siguientes alcances: 
- La evaluación del funcionamiento del prototipo ha sido validada para una sola empresa.

- El estudio no abarca la estimación de la demanda porque muchas empresas cuentan con sistemas para el desarrollo de sus pronósticos.

\section{Marco teórico}

\subsection{Sistema de soporte de decisiones}

Según Turban (1995), un sistema de soporte de decisiones se define de la siguiente forma:

Sistema de información basado en un computador interactivo, flexible y adaptable, especialmente desarrollado para apoyar la solución de un problema de gestión no estructurado para mejorar la toma de decisiones. Utiliza datos, proporciona una interfaz amigable y permite la toma de decisiones en el propio análisis de la situación.

Otra definición brindada por Eom y Kim (2006) es la siguiente:

Sistema de soporte de decisiones es definido como un sistema interactivo basado en computador que da soporte a los tomadores de decisiones en vez de reemplazarlos, utiliza datos y modelos para resolver problemas con diferentes grados de estructura: no estructurados, semiestructurados, tareas estructuradas y no estructuradas, semiestructuradas y no estructuradas y se enfoca más en la eficiencia de los procesos de decisión (facilita el proceso de decisión).

Esta última definición menciona que los sistemas de soporte de decisiones solucionan problemas con diferente grado de estructura (Turban, Aronson y Liang, 2005), tales como:

i. Decisión estructurada: Se resuelve mediante procesos específicos.

ii. Decisión no estructurada: La solución generada por el sistema de soporte decisiones sirve como conocimiento para tomar la decisión.

iii. Decisión semiestructurada: Se diferencia de una decisión estructurada porque cuenta con componentes de decisión humana.

Ramos, citado en Ruiz, Hernández y Giraldo (2009), lista los siguientes tipos como parte de sistemas informáticos para la toma de decisiones:

- $\quad$ Management Science (MS)

- $\quad$ Enterprise Resource Planning (ERP) 
- $\quad$ Business Analytics

- $\quad$ Customer Resource Management (CRM)

- Supply Chain Management (SCM)

- Data warehousing

- Knowledge Management System (KMS)

- Business Intelligence

- $\quad$ Expert Systems (ES)

- Online Analytical Processing

- $\quad$ Artificial Neural Network (ANN)

- Computer Assisted Engineering

- Intelligent Agents

- $\quad$ Group Support Systems

- Electronic Commerce DSS

- Enterprise Information System

- $\quad$ Enterprise Resource Management (ERM)

Se puede decir que los sistemas de soporte de decisiones tienen más características de un sistema de apoyo que de automatización de procesos. Dicho esto es relevante decir que en la organización un grupo pequeño de personas de, generalmente, alto nivel son las que manejan esta herramienta; este grupo se encarga de procesar los datos disponibles, generar las alternativas que permitan solucionar problemas y tomar decisiones. El sistema de soporte de decisiones tiene como tareas principales realizar una evaluación y comparación de posibles alternativas de solución, presentar una medición de los datos procesados y brindar diferentes esquemas de resultados. Cabe resaltar que los sistemas de soporte de decisiones se apoyan en el procesamiento de información por medio de un computador.

El concepto de apoyo a las decisiones ha evolucionado desde dos áreas principales de investigación: los estudios teóricos de organización de la toma de decisiones, hechos en el Carnegie Institute of Technology a finales de 1950 y comienzos de 1960, y el 
trabajo técnico sobre sistemas informáticos interactivos, principalmente elevados a cabo en el Instituto Tecnológico de Massachusetts en la década de 1960. Se considera que el concepto de dichos sistemas se convirtió en un espacio de investigación como tal alrededor de 1965, antes de ganar en intensidad durante la década de 1980. Para finales de dicho período, los sistemas de información ejecutiva (EIS), los sistemas de apoyo a la decisión en grupo (GDSS) y los sistemas organizacionales de apoyo a la decisión (ODSS) evolucionaron desde el usuario individual hasta los sistemas de soporte de decisiones orientados a modelos. A partir de 1990 aproximadamente, los almacenes de datos y el procesamiento analítico en línea (OLAP) comenzaron a ampliar el ámbito de los sistemas de soporte de decisiones. Con el cambio de milenio, se introdujeron nuevas aplicaciones analíticas basadas en la web (Keen y Scott, 1978).

\subsection{Programación no lineal}

La programación no lineal es una metodología para resolver problemas de optimización mediante un sistema de ecuaciones e inecuaciones, asociadas a un conjunto de restricciones y que presentan una función objetivo de maximización o minimización. La aplicación de esta metodología resulta compleja de modelar, el desarrollador tiene que conocer bien el caso del negocio para poder realizar un adecuado planeamiento del modelo; también debe estar en la capacidad de verificar si el modelo se ajusta a la realidad del negocio mediante una evaluación de los resultados obtenidos para distintos escenarios.

Existen diversos tipos de problemas de programación no lineal debido a la alta variabilidad que presentan muchos de ellos y por esto se emplean diferentes algoritmos de solución. En ciertas ocasiones las funciones presentan un planteamiento sencillo y los problemas pueden solucionarse eficientemente. En otros casos, inclusive problemas con una reducida cantidad de variables presentan un alto nivel de complejidad.

Cuando un problema de programación no lineal tiene a lo más dos variables, se puede representar en forma gráfica; de tal forma que las funciones no lineales representarán líneas curvas y si se tuvieran funciones lineales estas se presentarían con líneas rectas; por lo que la función objetivo y la región factible no tendrán el aspecto que adquieren en la programación lineal. La solución de este tipo de problemas no necesariamente tiene que estar en un vértice de la región factible o en la frontera de la misma. Además, en la programación no lineal un máximo local no necesariamente es un máximo global, y los algoritmos de búsqueda no pueden identificar cuando han hallado un máximo local o un máximo global. Por lo tanto, es importante conocer las condiciones en las que se define a un máximo local y a un máximo global en la región factible (Merino, 2015). 


\subsection{Revenue management}

El proceso de venta de un producto o servicio se enfrenta a tres interrogantes, la cantidad, el precio y cuándo vender, siempre resaltando la incertidumbre de la demanda. Para tomar acciones sobre esa demanda incierta es necesario para la organización segmentar a sus clientes y fijar un determinado precio a cada segmento, según el tipo de servicio que se le brindará.

Revenue management es la aplicación de herramientas de análisis para pronosticar el comportamiento de los clientes o consumidores de un servicio o producto, permitiendo la optimización del precio para maximizar la función objetivo definida. También se puede definir el revenue management como el proceso de entender, anticiparse e influenciar el comportamiento del consumidor con el fin de maximizar los ingresos esperados. Si bien la toma de decisiones de tipo revenue management existe desde la antigüedad, la novedad no son las decisiones en sí, sino el camino o forma como se llegan a tomar esas decisiones que gracias a la evolución del manejo de la información en nuestros tiempos ha ido mejorando.

El objetivo principal del revenue management es la venta del producto adecuado al cliente adecuado, en el momento adecuado, al precio correcto y en el canal correcto. La esencia de esta disciplina es la comprensión de la percepción del valor del producto para los clientes, alineándola con precisión a los precios de los productos, la colocación y disponibilidad para cada segmento de clientes. Todo esto soportado en una cultura organizacional basada en el análisis (Cross, 1997).

El crecimiento del uso de revenue management se ha incrementado en los últimos años gracias al avance tecnológico de la informática y los resultados obtenidos en distintas empresas, convirtiéndolas en casos de éxito. Los sectores que se han visto más favorecidos por el uso de revenue management son la hotelería y el turismo.

Recientemente, se observan actitudes más favorables a que tanto propietarios como gestores de establecimientos hoteleros apliquen estos sistemas para gestionar su inventario de servicios pero, aun así, el hotelero es uno de los sectores de la industria turística que ha adaptado más despacio sistemas de revenue management. (Mainzer, 2004)

Para obtener los resultados económicos por una buena gestión de revenue management en un tiempo prudencial es necesario contar con tres factores importantes antes de concretarse: el primero es el acceso a la información de forma oportuna, por ejemplo el conocimiento de las ofertas y precios de la competencia antes de tomar una decisión; el segundo factor es definir correctamente las temporadas de venta en el año; finalmente, el tercer factor es contar con un mecanismo de fijación de precios que permita establecer el precio exacto en el momento correcto (Yeoman, 2001). Por otra 
parte, Karadjov y Farahmand (2007) mencionan que para obtener una ventaja competitiva del uso de revenue management es necesario conocer y respetar las reglas de negocio antes de concretar una implementación. Es decir, que revenue management debe adaptarse al negocio y no al contrario.

Los modelos tradicionales de revenue management, que asumen información de demanda probabilística, son muy sensibles a errores en el método de predicción Weatherford y Belobaba (2002). En lugar de estimar una distribución de probabilidad de la demanda a partir de datos históricos, los métodos de optimización utilizan directamente datos históricos de ventas para optimizar los controles de reserva con un algoritmo de gradiente estocástico (Ryzin y McGill, 2000).

En entornos empresariales nuevos o no estacionarios, los datos históricos no están disponibles o son de poco valor. De hecho, la única información de demanda que esta dispone a menudo proviene de expertos de la industria, basados en su juicio y experiencia. Por lo tanto, las decisiones deben tomarse bajo limitada información de la demanda y modelos de optimización (Gallego, Ryan y Simchi-Levi, 2001).

\subsubsection{Proceso de definición de los elementos del sistema}

El conocimiento de las variables del negocio y su definición en el planteamiento de un modelo es el primer paso para implementar un sistema con revenue management, luego, es necesario categorizar estas variables y analizar cómo los cambios de cada categoría afectarían el precio final del producto o servicio, finalmente en base a las variables se logran obtener indicadores que medidos cuantitativamente sirven como retroalimentación para categorizar nuevamente las variables.

Figura 1. Niveles del proceso

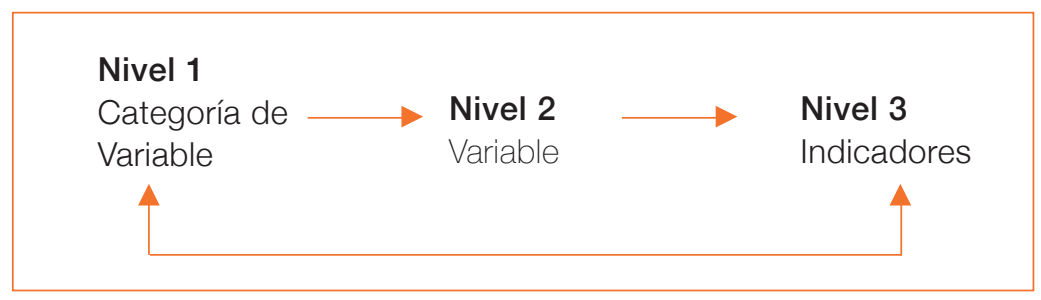

Se presentan los tres pasos para implementar un sistema con revenue management.

Fuente: Prats y Guia (2012, p. 513) 
Los elementos mencionados pueden clasificarse como estáticos o dinámicos. Los estáticos marcarán un precio mínimo y máximo en un periodo inicial y los dinámicos generarán la fluctuación dentro de esos límites.

\section{Figura 2. Fluctuación del precio del proceso}

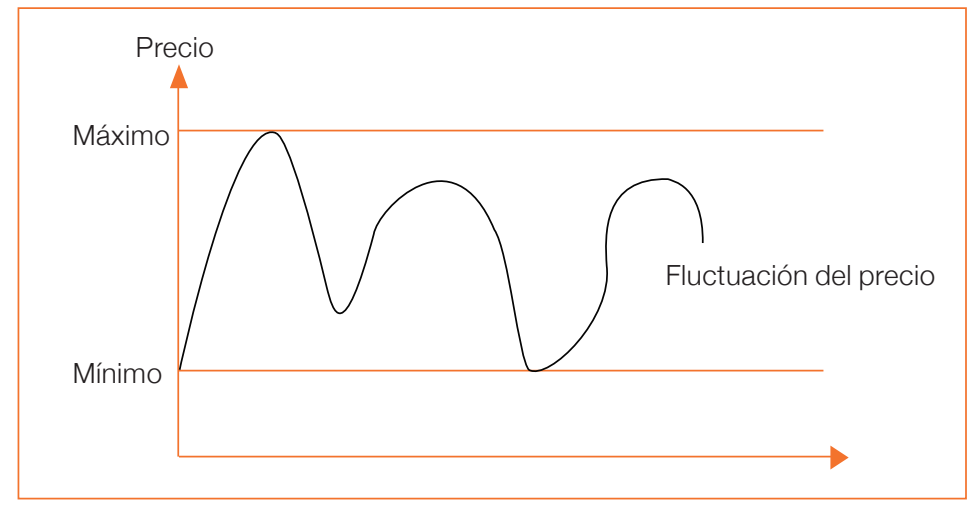

El gráfico muestra una representación de la fluctuación de precio.

Fuente: Prats y Guia (2012, p. 515)

\subsubsection{Sistema de establecimiento de precios (pricing)}

Las decisiones sobre la fijación de precios no solo son importantes sino que también son complejas (Steed y Gu, 2005). El reto consiste en disponer de un proceso de establecimiento de precios efectivo, que tenga en cuenta las necesidades de los clientes pero también las necesidades de los beneficiarios de los establecimientos, la integridad de la marca y los factores macroeconómicos (Vinod, 2004).

\subsubsection{Casos de revenue management}

El revenue management proviene originalmente de la industria aérea, específicamente de la compañía American Airlines, que fue la que inventó e implementó por primera vez este concepto. Forgacs (2010) menciona que esta disciplina empezó su extensión en las demás compañías aéreas en la década de los ochenta.

Para la gestión de la demanda, las aerolíneas habían utilizado a través de los años políticas de control de asientos como ofertas en los precios y reservas; el problema original era determinar los precios de los pasajes para las diferentes clases de vuelo sin perder potenciales clientes y mantener los ingresos deseados. Esto dio lugar a usar distintas técnicas de optimización como respuesta hasta el desarrollo de herramientas de revenue management; donde se utilizan distintos modelos 
de asignación de asientos, segmentación de clientes y fijación de precios para maximizar el beneficio (rentabilidad) esperado, sujetos a restricciones de tiempo, capacidad de los aviones en la red. Se volvió más conocido como network revenue management en este sector (Jaramillo, 2012).

En la industria de servicios tales como aerolíneas, transporte y hoteles, donde existe límite en la capacidad por espacio, se enfrentan con los problemas de asignación de precios y gestión de inventario. Al ser el servicio un producto perecible, el uso de revenue management permite afrontar estos problemas de decisión.

\section{Caso de análisis}

Para analizar a fondo esta problemática se contactó con la empresa terrestre interprovincial Ticllas S.A.C. con atención en Lima, Ayacucho, Huancavelica y Junín. Esta empresa, inició sus actividades con cuatro buses y solo atendía la ruta Huancavelica-Huancayo; después de recibir el apoyo de los familiares del fundador, quienes posteriormente se convertirían en socios, esta empresa empezó su crecimiento y actualmente dispone de una flota de cuarenta buses. Sin embargo, el crecimiento de la empresa reveló una falta de planificación de trabajo que, hasta el día de hoy, tratan de afrontar de la mejor manera.

En esta empresa la programación semanal de los viajes es realizada, de forma manual, por el área de administración; se utilizan los códigos de cada chofer para asignarlos al código de cada bus y programar las diferentes rutas y viajes. La regla actual de la programación se rige en asignar un número similar de salidas a cada bus para cada periodo. El proceso señalado presenta los siguientes inconvenientes:

- Es muy complicado, les toma mucho tiempo y presenta mayor riesgo de cometer errores.

- Se generan retrasos en la partida de los viajes, por ende una disminución en la calidad del servicio.

- Existen muchos cambios que se producen en la semana y esto genera trabajo extra debido a una reprogramación del itinerario.

- Se generan costos extra de operación de contratación y otros debido a la paralización de conductores y vehículos.

Antes de presentar el desarrollo de la solución es apropiado analizar los siguientes conceptos para entender mejor el caso de estudio: 


\section{Camino óptimo}

Es el mejor camino posible en un viaje, para obtener el mayor beneficio (costo mínimo o ganancia máxima) en un período de tiempo determinado, teniendo en cuenta una serie de condiciones (restricciones). Por ejemplo, si se transporta pasajeros por distintas ciudades y debe determinarse qué camino seguir para los próximos tres días, de tal forma que se obtenga la mayor ganancia, lo que se debe tener en cuenta son las siguientes condiciones: puede realizar solo un viaje por día y el bus parte de la ciudad de Lima. Las rutas son las siguientes:

Figura 3. Rutas posibles

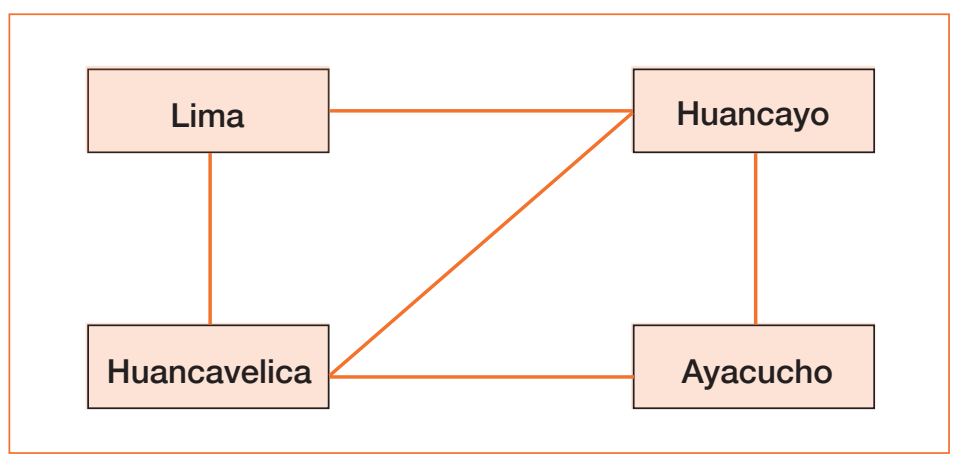

Elaboración propia

Las ganancias de cada ruta varían dependiendo de los días, según la siguiente tabla:

Tabla 2. Ganancia por ruta y día

\begin{tabular}{|c|c|}
\hline Día / Ruta & Ganancia \\
\hline \multicolumn{2}{|l|}{ Día 1} \\
\hline Lima-Huancayo & 300 \\
\hline Lima-Huancavelica & 350 \\
\hline \multicolumn{2}{|l|}{ Día 2} \\
\hline Huancayo-Huancavelica & 200 \\
\hline Huancayo-Lima & 250 \\
\hline Huancavelica-Lima & 300 \\
\hline Huancavelica-Ayacucho & 300 \\
\hline Huancavelica-Huancayo & 250 \\
\hline
\end{tabular}




\begin{tabular}{|c|c|}
\hline Día 3 & \\
\hline Huancavelica-Lima & 200 \\
\hline Huancavelica-Huancayo & 250 \\
\hline Huancavelica-Ayacucho & 300 \\
\hline Lima-Huancayo & 200 \\
\hline Lima-Huancavelica & 250 \\
\hline Ayacucho-Huancavelica & 300 \\
\hline Huancayo-Lima & 250 \\
\hline Huancayo-Huancavelica & 200 \\
\hline
\end{tabular}

Elaboración propia

Las posibles rutas para los próximos tres días son:

Figura 4. Alternativas de solución para identificar el camino óptimo.

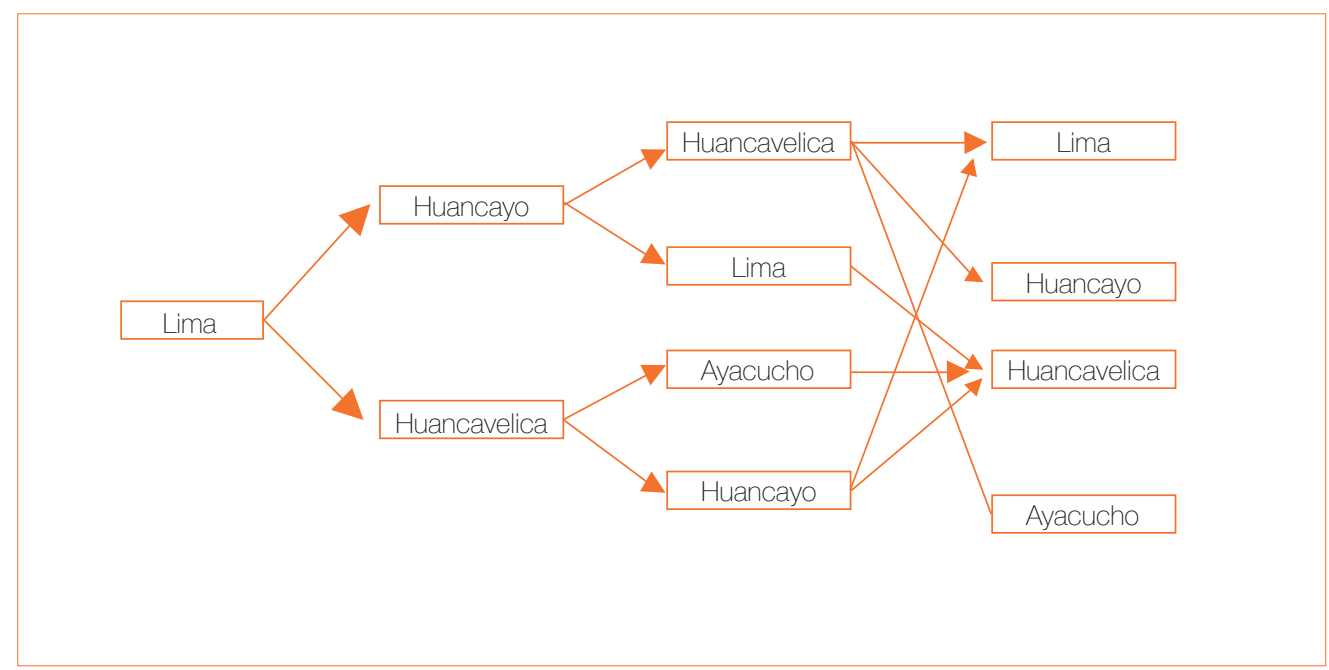

Elaboración propia 
Y las ganancias recaudadas a lo largo del período serían:

Tabla 3. Mejor opción y ganancia total para el primer caso

\begin{tabular}{|l|c|c|c|c|}
\hline \multicolumn{1}{|c|}{ Programación de rutas } & Día 1 & Día 2 & Día 3 & $\begin{array}{c}\text { Total } \\
\text { (S/) }\end{array}$ \\
\hline Lima-Huancayo-Huancavelica-Lima & 300 & 200 & 200 & 700 \\
\hline Lima-Huancayo-Huancavelica-Huancayo & 300 & 200 & 250 & 750 \\
\hline Lima-Huancayo-Huancavelica-Ayacucho & 300 & 200 & 300 & 800 \\
\hline Lima-Huancayo-Lima-Huancayo & 300 & 250 & 200 & 750 \\
\hline Lima-Huancayo-Lima-Huancavelica & 300 & 250 & 250 & 800 \\
\hline Lima-Huancavelica-Lima-Huancayo & 350 & 200 & 200 & 750 \\
\hline Lima-Huancavelica-Lima-Huancavelica & 350 & 200 & 250 & 800 \\
\hline Lima-Huancavelica-Ayacucho-Huancavelica & 350 & 300 & 300 & 950 \\
\hline Lima-Huancavelica-Huancayo-Lima & 350 & 250 & 250 & 850 \\
\hline Lima-Huancavelica-Huancayo-Huancavelica & 350 & 250 & 200 & 800 \\
\hline
\end{tabular}

Elaboración propia

Por lo tanto, el camino a seguir es Lima-Huancavelica-Ayacucho-Huancavelica para esperar recaudar S/ 950.

\section{Demanda y precio}

El mercado de transporte terrestre de pasajeros es complejo debido a que influyen varios factores para definir la demanda como "elástica" o "inelástica" en su relación precio-demanda. Por ejemplo, donde se tienen clientes de nivel socioeconómico B y C y el precio es un factor importante para que los clientes adquieran el servicio, una subida de los precios por parte de la empresa podría tener como consecuencia una variación considerable en su demanda de pasajeros. Sin embargo, si por temporada de fiestas o por aumento del precio del petróleo todo el grupo de empresas decide aumentar el precio de los pasajes, la demanda no se vería afectada pues no se cuentan con sustitutos.

En la investigación se han construido las herramientas necesarias para que la empresa pueda calcular el efecto de la variación de precios en distintos escenarios. Para profundizar más en el tema se plantea la siguiente situación: dos buses parten de la ciudad de Lima y se desea saber qué rutas deben escoger sus programadores y qué precio se le debe asignar al pasaje en los próximos dos días, para obtener el mayor ingreso. Pueden realizar solo un viaje por día y ambos buses tienen capacidad para cincuenta pasajeros. Las posibles rutas de viaje son las siguientes: 
Figura 5. Posibles rutas para determinar el recorrido y el mayor ingreso

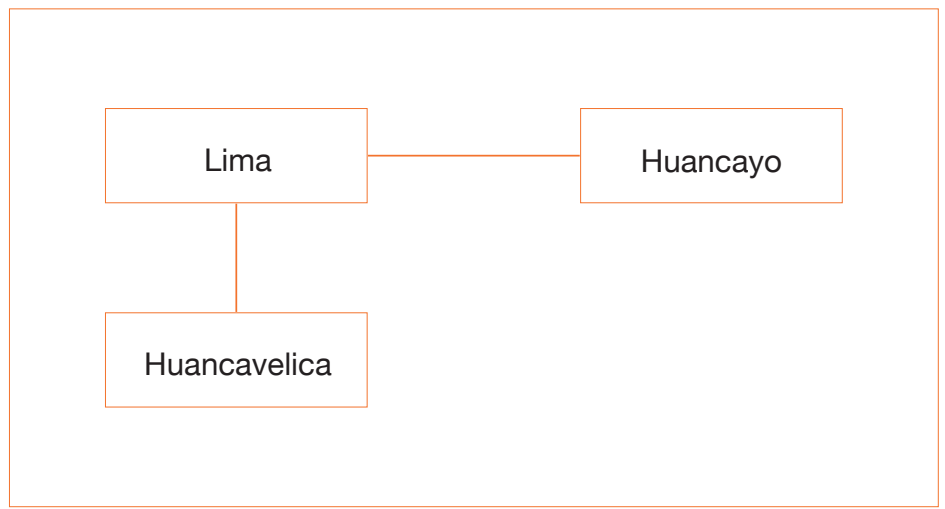

Elaboración propia

La relación demanda-precio para los próximos días es la siguiente:

Tabla 4. Relación demanda-precio para los destinos Lima, Huancayo y Huancavelica

\begin{tabular}{|l|r|r|}
\hline \multicolumn{1}{|c|}{ Día y ruta } & Precio del pasaje (S/) & Demanda de viajes \\
\hline Día 1 & 50 & 1 \\
\hline Lima-Huancayo & 40 & 2 \\
\hline Lima-Huancayo & 30 & 2 \\
\hline Lima-Huancavelica & 50 & 1 \\
\hline Lima-Huancavelica & & 2 \\
\hline Día 2 & 40 & 1 \\
\hline Huancayo-Lima & 50 & 2 \\
\hline Huancayo-Lima & 30 & 1 \\
\hline Huancavelica-Lima & 40 & \\
\hline Huancavelica-Lima & & \\
\hline
\end{tabular}

Elaboración propia 
Las posibles rutas para los próximos días son:

Figura 6. Posibles rutas entre los destinos Lima, Huancayo y Huancavelica

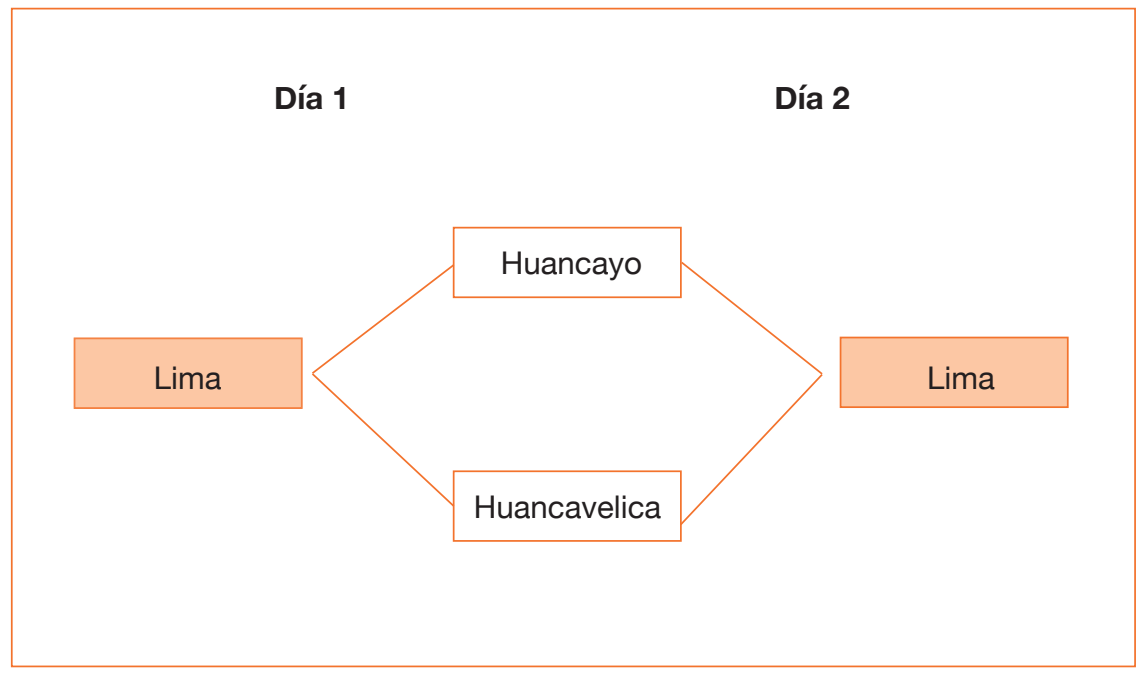

Elaboración propia

Tabla 5. Rutas e ingresos

\begin{tabular}{|c|c|c|c|c|}
\hline Día 1 & Día 2 & \multicolumn{3}{|c|}{ Total ingreso (S/) } \\
\hline $\begin{array}{l}\text { Bus A: Lima-Huancayo } \\
\text { Bus B: Lima-Huancavelica }\end{array}$ & $\begin{array}{l}\text { Bus A: Huancayo-Lima } \\
\text { Bus B:Huancavelica-Lima }\end{array}$ & $(50+50+50+40) * 50$ & $=$ & 9500 \\
\hline $\begin{array}{l}\text { Bus A: Lima-Huancayo } \\
\text { Bus B: Lima-Huancayo }\end{array}$ & $\begin{array}{l}\text { Bus A: Huancayo-Lima } \\
\text { Bus B: Huancayo-Lima }\end{array}$ & $(40+40+30+30)^{\star} 50$ & $=$ & 7000 \\
\hline $\begin{array}{l}\text { Bus A: Lima-Huancavelica } \\
\text { Bus B: Lima-Huancavelica }\end{array}$ & $\begin{array}{l}\text { Bus A: Huancavelica-Lima } \\
\text { Bus B: Huancavelica-Lima }\end{array}$ & $(30+30+30+30)^{\star} 50$ & $=$ & 6000 \\
\hline
\end{tabular}

Elaboración propia

Puede apreciarse que sería provechoso aumentar el precio y afrontar la disminución de demanda para obtener mayores ingresos. Esto siempre depende de las unidades disponibles, el horizonte de programación, las rutas y los valores de la relación entre el precio y la demanda. 


\section{Propuesta de solución}

\subsection{Enfoque}

Se analizaron distintos tipos de metodologías de optimización y se concluyó que se debería trabajar con un modelo de programación no lineal por los siguientes motivos:

- El caso de estudio se representa matemáticamente por un modelo no lineal y requiere de menor tiempo para ser modelado.

- La exactitud de los resultados, porque la programación no lineal es una metodología que puede encontrar exactamente el punto óptimo comparando varios resultados, detalle que, como se mencionó en el marco teórico de este trabajo, hace que supere en este punto a las metodologías heurísticas y metaheurísticas.

- El uso del revenue management en el proyecto servirá para proponer el precio de los pasajes según la demanda estimada de viajes.

\subsection{Desarrollo}

El modelo matemático busca construir un programa de viajes que obtenga la mayor rentabilidad posible.

\subsection{1. Índices}

Los datos y variables del modelo serán identificados mediante sus nombres correspondientes, así como con el uso de índices de identificación, los cuales se detallan a continuación:
d: Periodo de programación de viajes (día)
$d=1, \ldots, n 1$
i: Ciudad de origen
$i=1, \ldots, n 2$
j: Ciudad de destino
$j=1, \ldots, n 3$
b: Bus
$b=1, \ldots, n 4$
t: Índice de relación de precio y demanda
$t=1, \ldots, n 5$ 


\subsubsection{Datos}

Los datos de entrada para el modelo se detallan a continuación:

El(b, i): $\quad$ Bus "b" ubicado en la ciudad de origen "i" al inicio de la programación.

CTOVIAJE(i,j): Costo de viajar de la ciudad de origen "i" a la ciudad de destino "j".

DEMANDA(t, d, i, i): Demanda esperada de viajes para el periodo "d" desde la ciudad de origen "i" a la ciudad de destino "j" frente a un tipo de relación "t" con precio.

$\operatorname{PRECIO}(t, d, i, j)$ : Precio de los pasaje para el periodo "d" desde la ciudad de origen "i" a la ciudad de destino "j" frente a un tipo de relación "t" con demanda esperada.

CAPBUS(b): Capacidad de pasajeros del bus "b".

\subsubsection{Variables}

Los variables consideradas para el modelo son las siguientes:

PREDEM(t, $d, i, j): \quad 1$ Si se selecciona el tipo "t" de precio y demanda estimada el día "d" desde la ciudad de origen "i" a la ciudad de destino "j". O En caso contrario.

VIAJE $(d, b, i, j): \quad 1$ Si el bus "b" realiza un viaje con pasajeros el día "d" desde la ciudad de origen "i" a la ciudad de destino "j". O En caso contrario.

VIAJETEO(d,b,i,j): "i" a la ciudad de destino "j". O En caso contrario.

$\operatorname{DEMCAL}(\mathrm{d}, \mathrm{i}, \mathrm{j}): \quad \begin{aligned} & \text { Demanda seleccionada } \\ & \text { la ciudad de destino "j". }\end{aligned}$

PRECAL $(d, i, j)$ : $\quad$ Precio seleccionado de los pasajes para el día "d" desde la ciudad de origen "i" $\begin{array}{ll}\text { NUMVIAJE}(d, i, j): & \text { Número total de viajes con pasajeros realizados el día "d" desde la ciudad de } \\ & \text { origen "i" a la ciudad de destino "j". }\end{array}$

EID $(d, b, i): \quad 1$ Si el bus "b" se encuentra en la ciudad de origen "i" en el día " $d$ ". O En caso contrario. EF(d,b,j): $\quad \begin{array}{ll}1 \text { Si el bus "b" se encontrará en la ciudad de destino "J" en el día "d". O En caso } \\ \quad \text { contrario. }\end{array}$

\subsubsection{Función objetivo}

Se pretende maximizar la contribución marginal tratando de calcular los viajes necesarios que tiene que cumplir cada bus para cada ruta. Es así que, el precio de los pasajes multiplicado por la capacidad del bus para llevar pasajeros, nos da por resultado el ingreso estimado; al cual le restamos el costo de viaje y logramos obtener la utilidad correspondiente para cada ruta. 


$$
\sum_{d=1}^{n 1} \sum_{i=1}^{n 2} \sum_{j=1}^{n 3} \sum_{b=1}^{n 4} \begin{aligned}
& (\operatorname{PrECAL}(d, i, j) * \operatorname{CAPBUS}(b)-\operatorname{CtOVIAJE}(i, j)) * \operatorname{ViAJE}(d, b, i, j) \\
& -\operatorname{CtOVIAJE}(i, j) * \operatorname{ViajeteO}(d, b, i, j))
\end{aligned}
$$

\subsubsection{Restricciones:}

1. Cálculo del número total de viajes con pasajeros realizados el día "d" desde la ciudad de origen "i" a la ciudad de destino "j".

$$
\left.\sum_{b=1}^{n 4} \operatorname{VIAJE}(d, b, i, j)\right)=\operatorname{NUMMVIAJE}(d, i, j) \quad \forall d, \forall i, \forall j
$$

2. Selección de la demanda estimada del conjunto de tipos de relación con el precio seleccionado.

$$
\operatorname{DEMCAL}(d, i, j)=\sum_{t=1}^{n 5}\left(\operatorname{PREDEM}(t, d, i, j){ }^{*} \operatorname{DEMANDA}(t, d, i, j)\right) \quad \forall d, \forall i, \forall j
$$

3. Solo se puede seleccionar a lo más un tipo de relación entre demanda y precio para cada día y ruta.

$$
\sum_{t=1}^{n 5}(\operatorname{PREDEM}(t, d, i, j)) \leq 1 \quad \forall d, \forall i, \forall j
$$

4. El número de viajes a realizar debe ser menor o igual a la demanda de viajes estimada para cada ruta.

$$
\text { NUMVIAJE }(\mathrm{d}, \mathrm{i}, \mathrm{j}) \leq \operatorname{DEMCAL}(\mathrm{d}, \mathrm{i}, \mathrm{j}) \quad \forall \mathrm{d}, \forall i, \forall j
$$

5. Se define la ubicación de los buses en sus respectivas ciudades al iniciar la programación.

$$
E I D(1, b, i)=E l(b, i) \quad \forall b, \forall i
$$

6. Restricción para separar la realización de viaje con pasajeros y viajes de acomodo (sin pasajeros).

$$
\sum_{j=1}^{n 3} \operatorname{VIAJE}(\mathrm{d}, \mathrm{b}, \mathrm{i}, \mathrm{j})+\operatorname{VIAJETEO}(\mathrm{d}, \mathrm{b}, \mathrm{i}, \mathrm{j}) \leq 1 \quad \forall \mathrm{d}, \forall b, \forall i
$$


7. Los viajes realizados deben de partir desde la ciudad de origen en la frecuencia definida.

$$
\left.\sum_{j=1}^{n 3} \operatorname{VIAJE}(\mathrm{d}, \mathrm{b}, \mathrm{i}, \mathrm{j})+\operatorname{VIAJETEO}(\mathrm{d}, \mathrm{b}, \mathrm{i}, \mathrm{j})\right)=E I D(\mathrm{~d}, \mathrm{~b}, \mathrm{i}) \quad \forall \mathrm{d}, \forall \mathrm{b}, \forall i
$$

8. Los viajes realizados deben de llegar a su destino en la frecuencia definida

$$
\sum_{i=1}^{n 2} \operatorname{VIAJE}(\mathrm{d}, \mathrm{b}, \mathrm{i}, \mathrm{j})+\operatorname{VIAJETEO}(\mathrm{d}, \mathrm{b}, \mathrm{i}, \mathrm{j})=\operatorname{EF}(\mathrm{d}, \mathrm{b}, \mathrm{j}) \quad \forall \mathrm{d}, \forall b, \forall j
$$

9. Relación de igualdad entre la ciudad de origen y la ciudad destino para cada viaje, de cada bus, en la frecuencia definida.

$$
\begin{aligned}
& E I D(d, b, i)=E F(d-1, b, j) \quad \forall d, \forall b, \forall i \\
& d \geq 2, i=j
\end{aligned}
$$

10. Selección del precio del conjunto de tipos de relación con la demanda estimada seleccionada.

$$
\operatorname{PRECALd}, i, j=\sum_{t=1}^{n 5}\left(\operatorname{PREDEM}(\mathrm{t}, \mathrm{d}, \mathrm{i}, \mathrm{j}){ }^{*} \operatorname{PRECIO}(\mathrm{t}, \mathrm{d}, \mathrm{i}, \mathrm{j})\right) \quad \forall \mathrm{d}, \forall i, \forall j
$$

\subsubsection{Utilización del modelo}

Las condiciones para ejecutar el modelo son las siguientes:

Estado inicial

Antes de realizar la programación de los viajes se tiene en cuenta que los buses se encuentren distribuidos entre las diversas ciudades consideradas en las rutas, tal como se aprecia en la tabla 6.

\section{Tabla 6. Estado inicial de la programación: localización de las unidades}

\begin{tabular}{|c|l|}
\hline \multicolumn{1}{|c|}{ Bus } & Departamento \\
\hline A5H-957 & Lima \\
\hline B2J-951 & Huancavelica \\
\hline A8V-741 & Huancayo \\
\hline
\end{tabular}




\begin{tabular}{|c|c|}
\hline CJ-242 & Lima \\
\hline ROA-345 & Lima \\
\hline TS7-19 & Huancayo \\
\hline XMG-192 & Huancavelica \\
\hline$R S-456$ & Ayacucho \\
\hline AV-220 & Ayacucho \\
\hline DJ3-065 & Huancavelica \\
\hline R39-975 & Huancayo \\
\hline RT6-32 & Huancavelica \\
\hline D18-678 & Huancayo \\
\hline DA-251 & Ayacucho \\
\hline TA34-26 & Lima \\
\hline XL3-52 & Ayacucho \\
\hline XN6-521 & Lima \\
\hline B56-19 & Huancayo \\
\hline C26-55 & Ayacucho \\
\hline CA-456 & Huancavelica \\
\hline
\end{tabular}

Elaboración propia

Demanda y precios

Muestra todas las posibles rutas en el siguiente gráfico:

Figura 7. Rutas para la programación

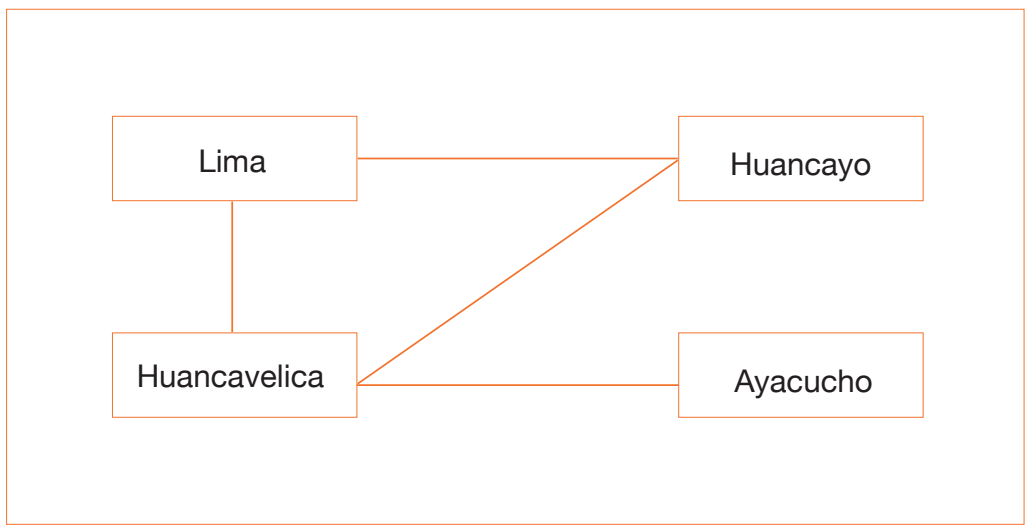

Elaboración propia 
El precio y la demanda tienen un aumento de 30 \% los fines de semana y feriados. Por otro lado, la relación precio-demanda para el uso de revenue management es que al aumentar 20 \% el precio de los pasajes, la demanda estimada bajará un 30 \%. De tal forma que para este escenario se cargaron los siguientes datos:

\section{Tabla 7. Datos de demanda y precio para la programación}

\begin{tabular}{|c|c|c|c|c|}
\hline Día & Origen & Destino & Demanda & Precio \\
\hline 01-nov-15 & Lima & Huancayo & 4 & 40 \\
\hline 01-nov-15 & Lima & Huancavelica & 3 & 50 \\
\hline 01-nov-15 & Huancayo & Lima & 4 & 40 \\
\hline 01-nov-15 & Huancayo & Huancavelica & 4 & 30 \\
\hline 01-nov-15 & Ayacucho & Huancavelica & 4 & 45 \\
\hline 01-nov-15 & Huancavelica & Lima & 4 & 45 \\
\hline 01-nov-15 & Huancavelica & Huancayo & 4 & 35 \\
\hline 01-nov-15 & Huancavelica & Ayacucho & 4 & 45 \\
\hline 02-nov-15 & Lima & Huancayo & 3 & 30 \\
\hline 02-nov-15 & Lima & Huancavelica & 2 & 40 \\
\hline 02-nov-15 & Huancayo & Lima & 3 & 30 \\
\hline 02-nov-15 & Huancayo & Huancavelica & 3 & 30 \\
\hline 02-nov-15 & Ayacucho & Huancavelica & 3 & 40 \\
\hline 02-nov-15 & Huancavelica & Lima & 3 & 30 \\
\hline 02-nov-15 & Huancavelica & Huancayo & 4 & 25 \\
\hline 02-nov-15 & Huancavelica & Ayacucho & 3 & 30 \\
\hline 03-nov-15 & Lima & Huancayo & 3 & 30 \\
\hline 03-nov-15 & Lima & Huancavelica & 2 & 40 \\
\hline 03-nov-15 & Huancayo & Lima & 3 & 30 \\
\hline 03-nov-15 & Huancayo & Huancavelica & 3 & 30 \\
\hline 03-nov-15 & Ayacucho & Huancavelica & 2 & 30 \\
\hline 03-nov-15 & Huancavelica & Lima & 3 & 30 \\
\hline 03-nov-15 & Huancavelica & Huancayo & 4 & 25 \\
\hline 03-nov-15 & Huancavelica & Ayacucho & 2 & 30 \\
\hline 04-nov-15 & Lima & Huancayo & 3 & 30 \\
\hline 04-nov-15 & Lima & Huancavelica & 3 & 40 \\
\hline 04-nov-15 & Huancayo & Lima & 3 & 30 \\
\hline 04-nov-15 & Huancayo & Huancavelica & 3 & 30 \\
\hline 04-nov-15 & Ayacucho & Huancavelica & 2 & 30 \\
\hline 04-nov-15 & Huancavelica & Lima & 3 & 35 \\
\hline 04-nov-15 & Huancavelica & Huancayo & 3 & 30 \\
\hline
\end{tabular}




\begin{tabular}{|c|c|c|c|c|}
\hline 04-nov-15 & Huancavelica & Ayacucho & 2 & 30 \\
\hline 05-nov-15 & Lima & Huancayo & 3 & 30 \\
\hline 05-nov-15 & Lima & Huancavelica & 3 & 40 \\
\hline 05-nov-15 & Huancayo & Lima & 3 & 30 \\
\hline 05-nov-15 & Huancayo & Huancavelica & 3 & 30 \\
\hline 05-nov-15 & Ayacucho & Huancavelica & 4 & 45 \\
\hline 05-nov-15 & Huancavelica & Lima & 3 & 30 \\
\hline 05-nov-15 & Huancavelica & Huancayo & 3 & 25 \\
\hline 05-nov-15 & Huancavelica & Ayacucho & 4 & 45 \\
\hline
\end{tabular}

Elaboración propia

Resultados de la ejecución del sistema

Para el prototipo se buscó solamente un óptimo local, por los límites de procesamiento que se tuvieron al hacer las pruebas. Arrojó la siguiente programación (se adjunta detalle de ingresos y costos por viaje):

Tabla 8. Resultados de programación

\begin{tabular}{|c|c|c|c|c|c|c|c|}
\hline Día & Bus & $\begin{array}{c}\text { DNI } \\
\text { Piloto }\end{array}$ & $\begin{array}{c}\text { DNI } \\
\text { Copiloto }\end{array}$ & Origen & Destino & $\begin{array}{l}\text { Ingreso } \\
(\mathrm{S} /)\end{array}$ & $\begin{array}{l}\text { Costo } \\
\text { (S/) }\end{array}$ \\
\hline 01-nov-15 & A5H-957 & 19968540 & 25843630 & Lima & Huancavelica & 3600 & 1372 \\
\hline 01-nov-15 & A8V-741 & 20007731 & 10018464 & Huancayo & Lima & 2500 & 1066 \\
\hline 01-nov-15 & AV-220 & 19948733 & 09015500 & Ayacucho & Ayacucho & 0 & 160 \\
\hline 01-nov-15 & B2J-951 & 19982336 & 08421166 & Huancavelica & Lima & 2750 & 1372 \\
\hline 01-nov-15 & B56-19 & 20046996 & 02690071 & Huancayo & Huancavelica & 1750 & 545 \\
\hline 01-nov-15 & C26-55 & 19997406 & 08636986 & Ayacucho & Huancavelica & 2750 & 1270 \\
\hline 01-nov-15 & CA-456 & 19962891 & 10777938 & Huancavelica & Huancayo & 2400 & 545 \\
\hline 01-nov-15 & CJ-242 & 19981010 & 25725674 & Lima & Huancayo & 2500 & 1066 \\
\hline 01-nov-15 & D18-678 & 19976880 & 10144128 & Huancayo & Lima & 3000 & 1066 \\
\hline 01-nov-15 & DA-251 & 72359665 & 25776709 & Ayacucho & Ayacucho & 0 & 160 \\
\hline 01-nov-15 & DJ3-065 & 08152149 & 31036946 & Huancavelica & Ayacucho & 2475 & 1270 \\
\hline 01-nov-15 & ROA-345 & 06771642 & 31602553 & Lima & Huancavelica & 3000 & 1372 \\
\hline 01-nov-15 & R39-975 & 25753085 & 04007364 & Huancayo & Huancavelica & 1750 & 545 \\
\hline 01-nov-15 & $\mathrm{RS}-456$ & 44909838 & 25835003 & Ayacucho & Huancavelica & 3300 & 1270 \\
\hline 01-nov-15 & RT6-32 & 43016812 & 07984894 & Huancavelica & Ayacucho & 3300 & 1270 \\
\hline 01-nov-15 & TA34-26 & 41874254 & 31043691 & Lima & Huancayo & 3000 & 1066 \\
\hline 01-nov-15 & TS7-19 & 42680323 & 25710466 & Huancayo & Lima & 3000 & 1066 \\
\hline $01-n o v-15$ & XL3-52 & 44333729 & 09933279 & Ayacucho & Huancavelica & 2750 & 1270 \\
\hline 01-nov-15 & XMG-192 & 07080588 & 21242061 & Huancavelica & Huancayo & 2000 & 545 \\
\hline 01-nov-15 & XN6-521 & 09651881 & 25774322 & Lima & Huancayo & 3000 & 1066 \\
\hline 02-nov-15 & $\mathrm{A} 5 \mathrm{H}-957$ & 19968540 & 25843630 & Huancavelica & Huancayo & 1800 & 545 \\
\hline
\end{tabular}




\begin{tabular}{|c|c|c|c|c|c|c|c|}
\hline 02-nov-15 & A8V-741 & 20007731 & 10018464 & Lima & Huancavelica & 2000 & 1372 \\
\hline 02-nov-15 & AV-220 & 19948733 & 09015500 & Ayacucho & Huancavelica & 2500 & 1270 \\
\hline 02-nov-15 & B2J-951 & 19982336 & 08421166 & Lima & Huancayo & 1750 & 1066 \\
\hline 02-nov-15 & B56-19 & 20046996 & 02690071 & Huancavelica & Huancavelica & 0 & 160 \\
\hline 02-nov-15 & C26-55 & 19997406 & 08636986 & Huancavelica & Huancayo & 1500 & 545 \\
\hline 02-nov-15 & CA-456 & 19962891 & 10777938 & Huancayo & Lima & 2100 & 1066 \\
\hline 02-nov-15 & CJ-242 & 19981010 & 25725674 & Huancayo & Lima & 1750 & 1066 \\
\hline 02-nov-15 & D18-678 & 19976880 & 10144128 & Lima & Huancayo & 2100 & 1066 \\
\hline 02-nov-15 & DA-251 & 72359665 & 25776709 & Ayacucho & Ayacucho & 0 & 160 \\
\hline 02-nov-15 & DJ3-065 & 08152149 & 31036946 & Ayacucho & Ayacucho & 0 & 160 \\
\hline 02-nov-15 & R0A-345 & 06771642 & 31602553 & Huancavelica & Huancayo & 1500 & 545 \\
\hline 02-nov-15 & R39-975 & 25753085 & 04007364 & Huancavelica & Lima & 1750 & 1372 \\
\hline 02-nov-15 & RS-456 & 44909838 & 25835003 & Huancavelica & Lima & 2100 & 1372 \\
\hline 02-nov-15 & RT6-32 & 43016812 & 07984894 & Ayacucho & Huancavelica & 3000 & 1270 \\
\hline 02-nov-15 & TA34-26 & 41874254 & 31043691 & Huancayo & Huancavelica & 1800 & 545 \\
\hline 02-nov-15 & TS7-19 & 42680323 & 25710466 & Lima & Huancavelica & 2400 & 1372 \\
\hline 02-nov-15 & XL3-52 & 44333729 & 09933279 & Huancavelica & Ayacucho & 1500 & 1270 \\
\hline 02-nov-15 & XMG-192 & 07080588 & 21242061 & Huancayo & Huancavelica & 1500 & 545 \\
\hline 02-nov-15 & XN6-521 & 09651881 & 25774322 & Huancayo & Huancavelica & 1800 & 545 \\
\hline 03-nov-15 & A5H-957 & 19968540 & 25843630 & Huancayo & Lima & 2100 & 1066 \\
\hline 03-nov-15 & A8V-741 & 20007731 & 10018464 & Huancavelica & Huancayo & 1500 & 545 \\
\hline 03-nov-15 & AV-220 & 19948733 & 09015500 & Huancavelica & Huancayo & 1500 & 545 \\
\hline 03-nov-15 & B2J-951 & 19982336 & 08421166 & Huancayo & Huancavelica & 1500 & 545 \\
\hline 03-nov-15 & B56-19 & 20046996 & 02690071 & Huancavelica & Lima & 1500 & 1372 \\
\hline 03-nov-15 & C26-55 & 19997406 & 08636986 & Huancayo & Lima & 1750 & 1066 \\
\hline 03-nov-15 & CA-456 & 19962891 & 10777938 & Lima & Huancavelica & 2400 & 1372 \\
\hline 03-nov-15 & CJ-242 & 19981010 & 25725674 & Lima & Huancavelica & 2000 & 1372 \\
\hline 03-nov-15 & D18-678 & 19976880 & 10144128 & Huancayo & Huancavelica & 1800 & 545 \\
\hline 03-nov-15 & DA-251 & 72359665 & 25776709 & Ayacucho & Huancavelica & 1500 & 1270 \\
\hline
\end{tabular}


Tabla 9. Resultados de programación

\begin{tabular}{|c|c|c|c|c|c|c|c|}
\hline Día & Bus & $\begin{array}{l}\text { DNI } \\
\text { Piloto }\end{array}$ & $\begin{array}{c}\text { DNI } \\
\text { Copiloto }\end{array}$ & Origen & Destino & $\begin{array}{l}\text { Ingreso } \\
(\mathrm{S} /)\end{array}$ & $\begin{array}{c}\text { Costo } \\
\text { (S/) }\end{array}$ \\
\hline 03-nov-15 & DJ3-065 & 08152149 & 31036946 & Ayacucho & Ayacucho & 0 & 160 \\
\hline 03-nov-15 & ROA-345 & 06771642 & 31602553 & Huancayo & Huancavelica & 1500 & 545 \\
\hline 03-nov-15 & R39-975 & 25753085 & 04007364 & Lima & Huancayo & 1750 & 1066 \\
\hline 03-nov-15 & RS-456 & 44909838 & 25835003 & Lima & Huancayo & 2100 & 1066 \\
\hline 03-nov-15 & RT6-32 & 43016812 & 07984894 & Huancavelica & Huancayo & 1800 & 545 \\
\hline 03-nov-15 & TA34-26 & 41874254 & 31043691 & Huancavelica & Ayacucho & 1800 & 1270 \\
\hline 03-nov-15 & TS7-19 & 42680323 & 25710466 & Huancavelica & Ayacucho & 1800 & 1270 \\
\hline 03-nov-15 & XL3-52 & 44333729 & 09933279 & Ayacucho & Huancavelica & 1500 & 1270 \\
\hline 03-nov-15 & XMG-192 & 07080588 & 21242061 & Huancavelica & Lima & 1500 & 1372 \\
\hline 03-nov-15 & XN6-521 & 09651881 & 25774322 & Huancavelica & Lima & 1800 & 1372 \\
\hline 04-nov-15 & A5H-957 & 19968540 & 25843630 & Lima & Huancavelica & 3000 & 1372 \\
\hline 04-nov-15 & A8V-741 & 20007731 & 10018464 & Huancayo & Huancavelica & 1500 & 545 \\
\hline 04-nov-15 & AV-220 & 19948733 & 09015500 & Huancayo & Huancavelica & 1500 & 545 \\
\hline 04-nov-15 & B2J-951 & 19982336 & 08421166 & Huancavelica & Huancayo & 1500 & 545 \\
\hline 04-nov-15 & B56-19 & 20046996 & 02690071 & Lima & Huancayo & 1500 & 1066 \\
\hline 04-nov-15 & C26-55 & 19997406 & 08636986 & Lima & Huancayo & 1500 & 1066 \\
\hline 04-nov-15 & CA-456 & 19962891 & 10777938 & Huancavelica & Lima & 2400 & 1372 \\
\hline 04-nov-15 & CJ-242 & 19981010 & 25725674 & Huancavelica & Huancavelica & 0 & 160 \\
\hline 04-nov-15 & D18-678 & 19976880 & 10144128 & Huancavelica & Lima & 2400 & 1372 \\
\hline 04-nov-15 & DA-251 & 72359665 & 25776709 & Huancavelica & Huancayo & 1500 & 545 \\
\hline 04-nov-15 & DJ3-065 & 08152149 & 31036946 & Ayacucho & Ayacucho & 0 & 160 \\
\hline 04-nov-15 & ROA-345 & 06771642 & 31602553 & Huancavelica & Ayacucho & 1750 & 1270 \\
\hline 04-nov-15 & R39-975 & 25753085 & 04007364 & Huancayo & Lima & 1750 & 1066 \\
\hline 04-nov-15 & RS-456 & 44909838 & 25835003 & Huancayo & Huancavelica & 1800 & 545 \\
\hline 04-nov-15 & RT6-32 & 43016812 & 07984894 & Huancayo & Lima & 2100 & 1066 \\
\hline 04-nov-15 & TA34-26 & 41874254 & 31043691 & Ayacucho & Huancavelica & 1800 & 1270 \\
\hline 04-nov-15 & TS7-19 & 42680323 & 25710466 & Ayacucho & Huancavelica & 1800 & 1270 \\
\hline 04-nov-15 & XL3-52 & 44333729 & 09933279 & Huancavelica & Huancayo & 1500 & 545 \\
\hline 04-nov-15 & XMG-192 & 07080588 & 21242061 & Lima & Huancayo & 1500 & 1066 \\
\hline 04-nov-15 & XN6-521 & 09651881 & 25774322 & Lima & Huancavelica & 3000 & 1372 \\
\hline 05-nov-15 & A5H-957 & 19968540 & 25843630 & Huancavelica & Lima & 2100 & 1372 \\
\hline 05-nov-15 & A8V-741 & 20007731 & 10018464 & Huancavelica & Huancayo & 1500 & 545 \\
\hline 05-nov-15 & AV-220 & 19948733 & 09015500 & Huancavelica & Huancayo & 1500 & 545 \\
\hline 05-nov-15 & B2J-951 & 19982336 & 08421166 & Huancayo & Huancavelica & 1500 & 545 \\
\hline 05-nov-15 & B56-19 & 20046996 & 02690071 & Huancayo & Lima & 1500 & 1066 \\
\hline
\end{tabular}




\begin{tabular}{|c|c|c|c|c|c|c|c|}
\hline 05-nov-15 & C26-55 & 19997406 & 08636986 & Huancayo & Lima & 1500 & 1066 \\
\hline 05-nov-15 & CA-456 & 19962891 & 10777938 & Lima & Huancavelica & 3000 & 1372 \\
\hline 05-nov-15 & CJ-242 & 19981010 & 25725674 & Huancavelica & Ayacucho & 2250 & 1270 \\
\hline 05-nov-15 & D18-678 & 19976880 & 10144128 & Lima & Huancayo & 2100 & 1066 \\
\hline 05-nov-15 & DA-251 & 72359665 & 25776709 & Huancayo & Lima & 1500 & 1066 \\
\hline 05-nov-15 & DJ3-065 & 08152149 & 31036946 & Ayacucho & Huancavelica & 2475 & 1270 \\
\hline 05-nov-15 & ROA-345 & 06771642 & 31602553 & Ayacucho & Huancavelica & 2750 & 1270 \\
\hline 05-nov-15 & R39-975 & 25753085 & 04007364 & Lima & Huancayo & 1750 & 1066 \\
\hline 05-nov-15 & RS-456 & 44909838 & 25835003 & Huancavelica & Ayacucho & 2700 & 1270 \\
\hline 05-nov-15 & RT6-32 & 43016812 & 07984894 & Lima & Huancavelica & 3000 & 1372 \\
\hline 05-nov-15 & TA34-26 & 41874254 & 31043691 & Huancavelica & Lima & 2100 & 1372 \\
\hline 05-nov-15 & TS7-19 & 42680323 & 25710466 & Huancavelica & Ayacucho & 2700 & 1270 \\
\hline 05-nov-15 & XL3-52 & 44333729 & 09933279 & Huancayo & Huancavelica & 1500 & 545 \\
\hline 05-nov-15 & XMG-192 & 07080588 & 21242061 & Huancayo & Huancavelica & 1500 & 545 \\
\hline 05-nov-15 & XN6-521 & 09651881 & 25774322 & Huancavelica & Ayacucho & 2700 & 1270 \\
\hline
\end{tabular}

Elaboración propia 
Figura 8. Programación de viajes para el día 1

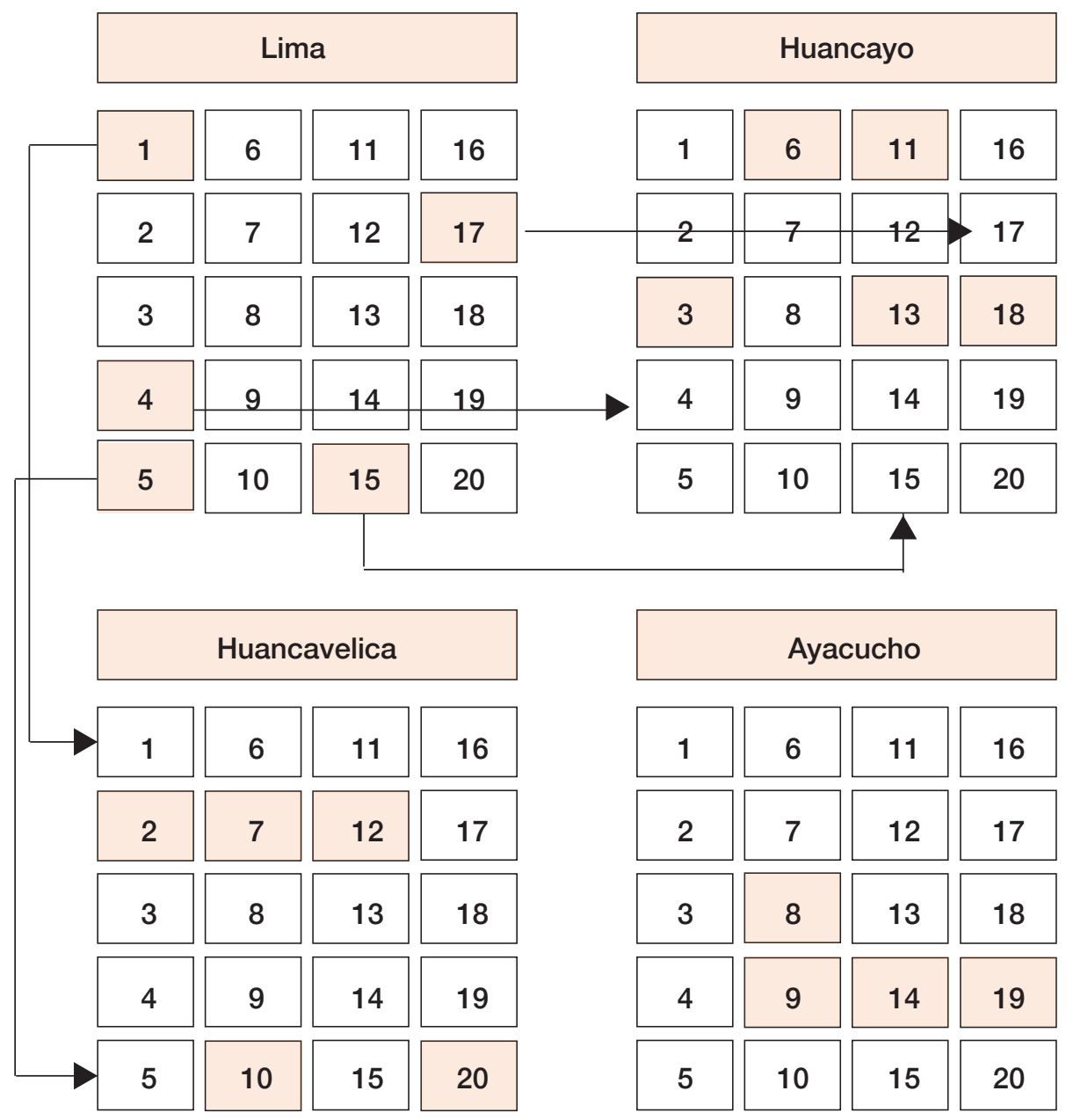

Elaboración propia

El ingreso total esperado es de S/190 200 y el costo total esperado de los viajes es de S/95 686, obteniendo así una contribución marginal de S/94 511. En cuanto a los precios de los pasajes, que podrán haberse modificado por el uso de revenue management, se descubrió que para la mayoría de casos es conveniente aumentar el precio y aceptar el costo de perder cierto porcentaje de su demanda quedando de la siguiente forma: 
Tabla 10. Resultados de programación

\begin{tabular}{|c|c|c|c|c|c|}
\hline Día & Origen & Destino & $\begin{array}{l}\text { Demanda } \\
\text { estimada }\end{array}$ & $\begin{array}{c}\text { Precio } \\
\text { propuesto }\end{array}$ & $\begin{array}{l}\text { Resultado } \\
\text { cantidad de } \\
\text { viajes }\end{array}$ \\
\hline 01-nov-15 & Lima & Huancayo & 3 & 50 & 3 \\
\hline 01-nov-15 & Lima & Huancavelica & 2 & 60 & 2 \\
\hline 01-nov-15 & Huancayo & Lima & 3 & 50 & 3 \\
\hline 01-nov-15 & Huancayo & Huancavelica & 3 & 35 & 2 \\
\hline 01-nov-15 & Ayacucho & Huancavelica & 3 & 55 & 3 \\
\hline 01-nov-15 & Huancavelica & Huancayo & 3 & 40 & 3 \\
\hline 01-nov-15 & Huancavelica & Ayacucho & 3 & 55 & 2 \\
\hline 02-nov-15 & Lima & Huancayo & 2 & 35 & 1 \\
\hline 02-nov-15 & Lima & Huancavelica & 1 & 50 & 1 \\
\hline 02-nov-15 & Huancayo & Lima & 2 & 35 & 2 \\
\hline 02-nov-15 & Huancayo & Huancavelica & 3 & 30 & 3 \\
\hline 02-nov-15 & Ayacucho & Huancavelica & 2 & 50 & 2 \\
\hline 02-nov-15 & Huancavelica & Lima & 2 & 35 & 1 \\
\hline 02-nov-15 & Huancavelica & Huancayo & 4 & 25 & 4 \\
\hline 03-nov-15 & Lima & Huancayo & 2 & 35 & 2 \\
\hline 03-nov-15 & Lima & Huancavelica & 1 & 50 & 1 \\
\hline 03-nov-15 & Huancayo & Lima & 2 & 35 & 2 \\
\hline 03-nov-15 & Huancayo & Huancavelica & 2 & 35 & 2 \\
\hline 03-nov-15 & Huancavelica & Huancayo & 3 & 30 & 3 \\
\hline 04-nov-15 & Lima & Huancayo & 2 & 35 & 1 \\
\hline 04-nov-15 & Lima & Huancavelica & 2 & 50 & 2 \\
\hline 04-nov-15 & Huancayo & Lima & 2 & 35 & 2 \\
\hline 04-nov-15 & Huancayo & Huancavelica & 3 & 30 & 3 \\
\hline 04-nov-15 & Huancavelica & Huancayo & 3 & 30 & 3 \\
\hline 04-nov-15 & Huancavelica & Ayacucho & 1 & 35 & 1 \\
\hline 05-nov-15 & Lima & Huancayo & 2 & 50 & 2 \\
\hline 05-nov-15 & Huancayo & Lima & 2 & 35 & 2 \\
\hline 05-nov-15 & Huancayo & Huancavelica & 3 & 30 & 3 \\
\hline 05-nov-15 & Ayacucho & Huancavelica & 3 & 55 & 3 \\
\hline 05-nov-15 & Huancavelica & Huancayo & 3 & 25 & 3 \\
\hline 05-nov-15 & Huancavelica & Ayacucho & 3 & 55 & 3 \\
\hline
\end{tabular}

Elaboración propia 
De tal forma, se puede observar que el número total de viajes con pasajeros en ese período fue de 92, repartidos de la siguiente manera:

\section{Tabla 11. Resultados de programación total de viajes}

\begin{tabular}{|r|l|c|c|c|c|c|}
\hline & \multicolumn{9}{|c|}{ Destino } \\
\hline \multirow{3}{*}{ Origen } & Ayacucho & 0 & Huancavelica & Huancayo & Lima & Total \\
\cline { 2 - 8 } & Huancavelica & 10 & 0 & 0 & 0 & 11 \\
\cline { 2 - 8 } & Huancayo & 0 & 14 & 0 & 13 & 33 \\
\cline { 2 - 9 } & Lima & 0 & 10 & 12 & 0 & 22 \\
\hline
\end{tabular}

Elaboración propia

Figura 9. Resultados de total de viajes

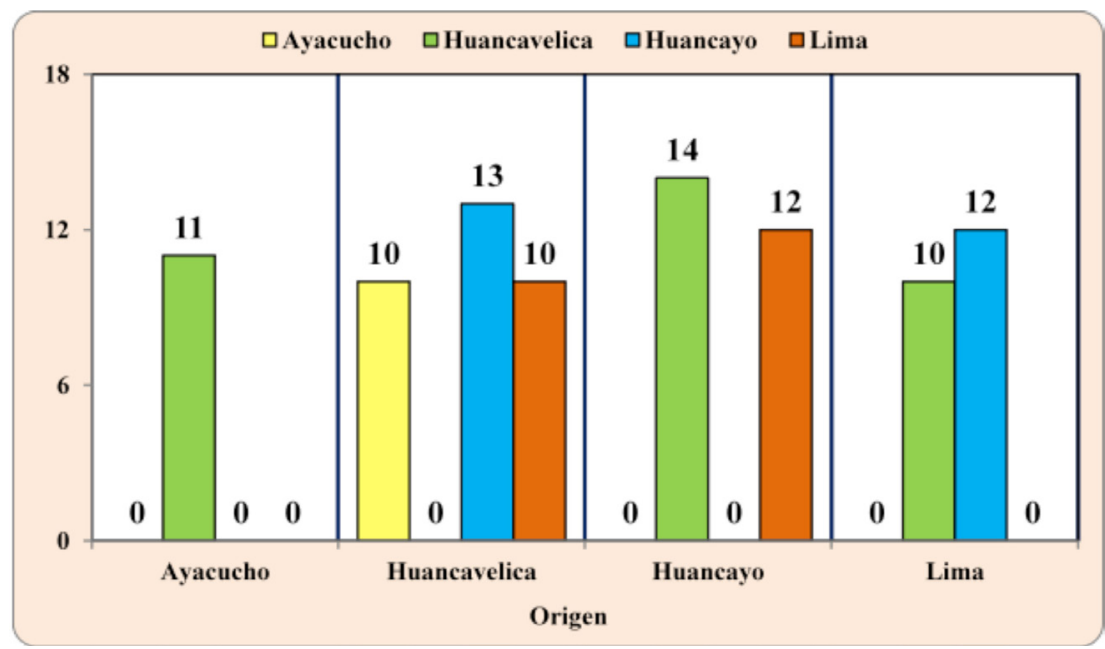

Elaboración propia

Resultados de una programación armada por la empresa

Gracias al apoyo del señor Josep Rocca, supervisor de la programación de viajes, se implementó un escenario de simulación con los veinte buses corridos en el ejemplo para el prototipo y el resultado fue lo siguiente: 
Tabla 12. Resultados de programación

\begin{tabular}{|c|c|c|c|c|c|c|c|}
\hline Día & Bus & $\begin{array}{l}\text { DNI } \\
\text { Piloto }\end{array}$ & $\begin{array}{c}\text { DNI } \\
\text { Copiloto }\end{array}$ & Origen & Destino & $\begin{array}{l}\text { Ingreso } \\
(\mathrm{S} /)\end{array}$ & $\begin{array}{l}\text { Costo } \\
\text { (S/) }\end{array}$ \\
\hline 01-nov-15 & A5H-957 & 19968540 & 25843630 & Lima & Huancayo & 2400 & 1066 \\
\hline 01-nov-15 & A8V-741 & 20007731 & 10018464 & Huancayo & Huancavelica & 1500 & 545 \\
\hline 01-nov-15 & AV-220 & 19948733 & 09015500 & Ayacucho & Ayacucho & 0 & 160 \\
\hline 01-nov-15 & B2J-951 & 19982336 & 08421166 & Huancavelica & Huancayo & 1750 & 545 \\
\hline 01-nov-15 & B56-19 & 20046996 & 02690071 & Huancayo & Huancavelica & 1500 & 545 \\
\hline 01-nov-15 & C26-55 & 19997406 & 08636986 & Ayacucho & Huancavelica & 2250 & 1270 \\
\hline 01-nov-15 & CA-456 & 19962891 & 10777938 & Huancavelica & Huancayo & 2100 & 545 \\
\hline 01-nov-15 & CJ-242 & 19981010 & 25725674 & Lima & Huancayo & 2000 & 1066 \\
\hline 01-nov-15 & D18-678 & 19976880 & 10144128 & Huancayo & Huancavelica & 1800 & 545 \\
\hline 01-nov-15 & DA-251 & 72359665 & 25776709 & Ayacucho & Ayacucho & 0 & 160 \\
\hline 01-nov-15 & DJ3-065 & 08152149 & 31036946 & Huancavelica & Lima & 2025 & 1372 \\
\hline 01-nov-15 & R0A-345 & 06771642 & 31602553 & Lima & Huancavelica & 2500 & 1372 \\
\hline 01-nov-15 & R39-975 & 25753085 & 04007364 & Huancayo & Lima & 2000 & 1066 \\
\hline 01-nov-15 & RS-456 & 44909838 & 25835003 & Ayacucho & Huancavelica & 2700 & 1270 \\
\hline 01-nov-15 & RT6-32 & 43016812 & 07984894 & Huancavelica & Lima & 2700 & 1372 \\
\hline 01-nov-15 & TA34-26 & 41874254 & 31043691 & Lima & Huancavelica & 3000 & 1372 \\
\hline 01-nov-15 & TS7-19 & 42680323 & 25710466 & Huancayo & Lima & 2400 & 1066 \\
\hline 01-nov-15 & XL3-52 & 44333729 & 09933279 & Ayacucho & Ayacucho & 0 & 160 \\
\hline 01-nov-15 & XMG-192 & 07080588 & 21242061 & Huancavelica & Ayacucho & 2250 & 1270 \\
\hline 01-nov-15 & XN6-521 & 09651881 & 25774322 & Lima & Huancayo & 2400 & 1066 \\
\hline 02-nov-15 & A5H-957 & 19968540 & 25843630 & Huancayo & Lima & 1800 & 1066 \\
\hline 02-nov-15 & A8V-741 & 20007731 & 10018464 & Huancavelica & Ayacucho & 1500 & 1270 \\
\hline 02-nov-15 & AV-220 & 19948733 & 09015500 & Ayacucho & Huancavelica & 2000 & 1270 \\
\hline 02-nov-15 & B2J-951 & 19982336 & 08421166 & Huancayo & Lima & 1500 & 1066 \\
\hline 02-nov-15 & B56-19 & 20046996 & 02690071 & Huancavelica & Lima & 1500 & 1372 \\
\hline 02-nov-15 & C26-55 & 19997406 & 08636986 & Huancavelica & Lima & 1500 & 1372 \\
\hline 02-nov-15 & CA-456 & 19962891 & 10777938 & Huancayo & Huancavelica & 1800 & 545 \\
\hline 02-nov-15 & CJ-242 & 19981010 & 25725674 & Huancayo & Huancavelica & 1500 & 545 \\
\hline 02-nov-15 & D18-678 & 19976880 & 10144128 & Huancavelica & Lima & 1800 & 1372 \\
\hline 02-nov-15 & DA-251 & 72359665 & 25776709 & Ayacucho & Ayacucho & 0 & 160 \\
\hline 02-nov-15 & DJ3-065 & 08152149 & 31036946 & Lima & Huancayo & 1350 & 1066 \\
\hline 02-nov-15 & R0A-345 & 06771642 & 31602553 & Huancavelica & Huancayo & 1250 & 545 \\
\hline 02-nov-15 & R39-975 & 25753085 & 04007364 & Lima & Huancayo & 1500 & 1066 \\
\hline 02-nov-15 & RS-456 & 44909838 & 25835003 & Huancavelica & Huancayo & 1500 & 545 \\
\hline 02-nov-15 & RT6-32 & 43016812 & 07984894 & Lima & Huancavelica & 2400 & 1372 \\
\hline
\end{tabular}




\begin{tabular}{|c|c|c|c|c|c|c|c|}
\hline 02-nov-15 & TA34-26 & 41874254 & 31043691 & Huancavelica & Huancayo & 1500 & 545 \\
\hline 02-nov-15 & TS7-19 & 42680323 & 25710466 & Lima & Huancavelica & 2400 & 1372 \\
\hline 02-nov-15 & XL3-52 & 44333729 & 09933279 & Ayacucho & Huancavelica & 2000 & 1270 \\
\hline 02-nov-15 & XMG-192 & 07080588 & 21242061 & Ayacucho & Ayacucho & 0 & 160 \\
\hline 02-nov-15 & XN6-521 & 09651881 & 25774322 & Huancayo & Huancavelica & 1800 & 545 \\
\hline 03-nov-15 & A5H-957 & 19968540 & 25843630 & Lima & Huancayo & 1800 & 1066 \\
\hline 03-nov-15 & A8V-741 & 20007731 & 10018464 & Ayacucho & Ayacucho & 0 & 160 \\
\hline 03-nov-15 & AV-220 & 19948733 & 09015500 & Huancavelica & Ayacucho & 1500 & 1270 \\
\hline 03-nov-15 & B2J-951 & 19982336 & 08421166 & Lima & Huancavelica & 2000 & 1372 \\
\hline 03-nov-15 & B56-19 & 20046996 & 02690071 & Lima & Huancayo & 1500 & 1066 \\
\hline 03-nov-15 & C26-55 & 19997406 & 08636986 & Lima & Huancavelica & 2000 & 1372 \\
\hline 03-nov-15 & CA-456 & 19962891 & 10777938 & Huancavelica & Huancayo & 1500 & 545 \\
\hline 03-nov-15 & CJ-242 & 19981010 & 25725674 & Huancavelica & Huancayo & 1250 & 545 \\
\hline 03-nov-15 & D18-678 & 19976880 & 10144128 & Lima & Huancayo & 1800 & 1066 \\
\hline 03-nov-15 & DA-251 & 72359665 & 25776709 & Ayacucho & Huancavelica & 1500 & 1270 \\
\hline
\end{tabular}

Elaboración propia

Tabla 13. Resultados de programación

\begin{tabular}{|c|c|c|c|c|c|c|c|}
\hline Día & Bus & $\begin{array}{l}\text { DNI } \\
\text { Piloto }\end{array}$ & $\begin{array}{c}\text { DNI } \\
\text { Copiloto }\end{array}$ & Origen & Destino & $\begin{array}{l}\text { Ingreso } \\
(\mathrm{S} /)\end{array}$ & $\begin{array}{l}\text { Costo } \\
\text { (S/) }\end{array}$ \\
\hline 03-nov-15 & DJ3-065 & 08152149 & 31036946 & Huancayo & Lima & 1350 & 1066 \\
\hline 03-nov-15 & ROA-345 & 06771642 & 31602553 & Huancayo & Huancavelica & 1500 & 545 \\
\hline 03-nov-15 & R39-975 & 25753085 & 04007364 & Huancayo & Lima & 1500 & 1066 \\
\hline 03-nov-15 & RS-456 & 44909838 & 25835003 & Huancayo & Huancavelica & 1800 & 545 \\
\hline 03-nov-15 & RT6-32 & 43016812 & 07984894 & Huancavelica & Huancayo & 1500 & 545 \\
\hline 03-nov-15 & TA34-26 & 41874254 & 31043691 & Huancayo & Huancavelica & 1800 & 545 \\
\hline 03-nov-15 & TS7-19 & 42680323 & 25710466 & Huancavelica & Lima & 1800 & 1372 \\
\hline 03-nov-15 & XL3-52 & 44333729 & 09933279 & Huancavelica & Lima & 1500 & 1372 \\
\hline 03-nov-15 & XMG-192 & 07080588 & 21242061 & Ayacucho & Huancavelica & 1500 & 1270 \\
\hline 03-nov-15 & XN6-521 & 09651881 & 25774322 & Huancavelica & Lima & 1800 & 1372 \\
\hline 04-nov-15 & A5H-957 & 19968540 & 25843630 & Huancayo & Lima & 1800 & 1066 \\
\hline 04-nov-15 & A8V-741 & 20007731 & 10018464 & Ayacucho & Ayacucho & 0 & 160 \\
\hline 04-nov-15 & AV-220 & 19948733 & 09015500 & Ayacucho & Huancavelica & 1500 & 1270 \\
\hline 04-nov-15 & B2J-951 & 19982336 & 08421166 & Huancavelica & Ayacucho & 1500 & 1270 \\
\hline 04-nov-15 & B56-19 & 20046996 & 02690071 & Huancayo & Lima & 1500 & 1066 \\
\hline 04-nov-15 & C26-55 & 19997406 & 08636986 & Huancavelica & Huancayo & 1500 & 545 \\
\hline 04-nov-15 & CA-456 & 19962891 & 10777938 & Huancayo & Lima & 1800 & 1066 \\
\hline
\end{tabular}




\begin{tabular}{|c|c|c|c|c|c|c|c|}
\hline 04-nov-15 & CJ-242 & 19981010 & 25725674 & Huancayo & Huancavelica & 1500 & 545 \\
\hline 04-nov-15 & D18-678 & 19976880 & 10144128 & Huancayo & Huancavelica & 1800 & 545 \\
\hline 04-nov-15 & DA-251 & 72359665 & 25776709 & Huancavelica & Huancayo & 1500 & 545 \\
\hline 04-nov-15 & DJ3-065 & 08152149 & 31036946 & Lima & Huancayo & 1350 & 1066 \\
\hline 04-nov-15 & ROA-345 & 06771642 & 31602553 & Huancavelica & Huancayo & 1500 & 545 \\
\hline 04-nov-15 & R39-975 & 25753085 & 04007364 & Lima & Huancayo & 1500 & 1066 \\
\hline 04-nov-15 & RS-456 & 44909838 & 25835003 & Huancavelica & Lima & 2100 & 1372 \\
\hline 04-nov-15 & RT6-32 & 43016812 & 07984894 & Huancayo & Huancavelica & 1800 & 545 \\
\hline 04-nov-15 & TA34-26 & 41874254 & 31043691 & Huancavelica & Lima & 2100 & 1372 \\
\hline 04-nov-15 & TS7-19 & 42680323 & 25710466 & Lima & Huancayo & 1800 & 1066 \\
\hline 04-nov-15 & XL3-52 & 44333729 & 09933279 & Lima & Huancavelica & 2000 & 1372 \\
\hline 04-nov-15 & XMG-192 & 07080588 & 21242061 & Huancavelica & Lima & 1750 & 1372 \\
\hline 04-nov-15 & XN6-521 & 09651881 & 25774322 & Lima & Huancavelica & 2400 & 1372 \\
\hline 05-nov-15 & $\mathrm{A} 5 \mathrm{H}-957$ & 19968540 & 25843630 & Lima & Huancayo & 1800 & 1066 \\
\hline 05-nov-15 & A8V-741 & 20007731 & 10018464 & Ayacucho & Huancavelica & 2250 & 1270 \\
\hline 05-nov-15 & AV-220 & 19948733 & 09015500 & Huancavelica & Lima & 1500 & 1372 \\
\hline 05-nov-15 & B2J-951 & 19982336 & 08421166 & Ayacucho & Huancavelica & 2250 & 1270 \\
\hline 05-nov-15 & B56-19 & 20046996 & 02690071 & Lima & Huancayo & 1500 & 1066 \\
\hline 05-nov-15 & C26-55 & 19997406 & 08636986 & Huancayo & Lima & 1500 & 1066 \\
\hline 05-nov-15 & CA-456 & 19962891 & 10777938 & Lima & Huancayo & 1800 & 1066 \\
\hline 05-nov-15 & CJ-242 & 19981010 & 25725674 & Huancavelica & Lima & 1500 & 1372 \\
\hline 05-nov-15 & D18-678 & 19976880 & 10144128 & Huancavelica & Huancayo & 1500 & 545 \\
\hline 05-nov-15 & DA-251 & 72359665 & 25776709 & Huancayo & Lima & 1500 & 1066 \\
\hline 05-nov-15 & DJ3-065 & 08152149 & 31036946 & Huancayo & Lima & 1350 & 1066 \\
\hline 05-nov-15 & R0A-345 & 06771642 & 31602553 & Huancayo & Huancavelica & 1500 & 545 \\
\hline 05-nov-15 & R39-975 & 25753085 & 04007364 & Huancayo & Huancavelica & 1500 & 545 \\
\hline 05-nov-15 & RS-456 & 44909838 & 25835003 & Lima & Huancavelica & 2400 & 1372 \\
\hline 05-nov-15 & RT6-32 & 43016812 & 07984894 & Huancavelica & Huancayo & 1500 & 545 \\
\hline 05-nov-15 & TA34-26 & 41874254 & 31043691 & Lima & Huancavelica & 2400 & 1372 \\
\hline 05-nov-15 & TS7-19 & 42680323 & 25710466 & Huancayo & Huancavelica & 1800 & 545 \\
\hline 05-nov-15 & XL3-52 & 44333729 & 09933279 & Huancavelica & Huancayo & 1250 & 545 \\
\hline 05-nov-15 & XMG-192 & 07080588 & 21242061 & Lima & Huancavelica & 2000 & 1372 \\
\hline 05-nov-15 & XN6-521 & 09651881 & 25774322 & Huancavelica & Ayacucho & 2700 & 1270 \\
\hline
\end{tabular}

Elaboración propia

El ingreso total esperado es de S/166 975 y el costo total esperado de los viajes es de S/95 349, obteniendo así una contribución marginal de S/71 626.

La demanda estimada y precio con que se armó la programación fueron: 
Tabla 14. Resultados de programación

\begin{tabular}{|c|c|c|c|c|c|}
\hline Día & Origen & Destino & $\mathrm{N}^{\circ}$ de viajes & Demanda & Precio (S/) \\
\hline 01-nov-15 & Ayacucho & Huancavelica & 2 & 4 & 45 \\
\hline 01-nov-15 & Huancavelica & Ayacucho & 1 & 4 & 45 \\
\hline 01-nov-15 & Huancavelica & Huancayo & 2 & 4 & 35 \\
\hline 01-nov-15 & Huancavelica & Lima & 2 & 4 & 45 \\
\hline 01-nov-15 & Huancayo & Huancavelica & 3 & 4 & 30 \\
\hline 01-nov-15 & Huancayo & Lima & 2 & 4 & 40 \\
\hline 01-nov-15 & Lima & Huancavelica & 2 & 3 & 50 \\
\hline 01-nov-15 & Lima & Huancayo & 3 & 4 & 40 \\
\hline 02-nov-15 & Ayacucho & Huancavelica & 2 & 3 & 40 \\
\hline 02-nov-15 & Huancavelica & Ayacucho & 1 & 3 & 30 \\
\hline 02-nov-15 & Huancavelica & Huancayo & 3 & 4 & 25 \\
\hline 02-nov-15 & Huancavelica & Lima & 3 & 3 & 30 \\
\hline 02-nov-15 & Huancayo & Huancavelica & 3 & 3 & 30 \\
\hline 02-nov-15 & Huancayo & Lima & 2 & 3 & 30 \\
\hline 02-nov-15 & Lima & Huancavelica & 2 & 2 & 40 \\
\hline 02-nov-15 & Lima & Huancayo & 2 & 3 & 30 \\
\hline 03-nov-15 & Ayacucho & Huancavelica & 2 & 2 & 30 \\
\hline 03-nov-15 & Huancavelica & Ayacucho & 1 & 2 & 30 \\
\hline 03-nov-15 & Huancavelica & Huancayo & 3 & 4 & 25 \\
\hline 03-nov-15 & Huancavelica & Lima & 3 & 3 & 30 \\
\hline 03-nov-15 & Huancayo & Huancavelica & 3 & 3 & 30 \\
\hline 03-nov-15 & Huancayo & Lima & 2 & 3 & 30 \\
\hline 03-nov-15 & Lima & Huancavelica & 2 & 2 & 40 \\
\hline 03-nov-15 & Lima & Huancayo & 3 & 3 & 30 \\
\hline 04-nov-15 & Ayacucho & Huancavelica & 1 & 2 & 30 \\
\hline 04-nov-15 & Huancavelica & Ayacucho & 1 & 2 & 30 \\
\hline 04-nov-15 & Huancavelica & Huancayo & 3 & 3 & 30 \\
\hline 04-nov-15 & Huancavelica & Lima & 3 & 3 & 35 \\
\hline 04-nov-15 & Huancayo & Huancavelica & 3 & 3 & 30 \\
\hline 04-nov-15 & Huancayo & Lima & 3 & 3 & 30 \\
\hline 04-nov-15 & Lima & Huancavelica & 2 & 3 & 40 \\
\hline 04-nov-15 & Lima & Huancayo & 3 & 3 & 30 \\
\hline 05-nov-15 & Ayacucho & Huancavelica & 2 & 4 & 45 \\
\hline 05-nov-15 & Huancavelica & Ayacucho & 1 & 4 & 45 \\
\hline 05-nov-15 & Huancavelica & Huancayo & 3 & 3 & 25 \\
\hline
\end{tabular}




\begin{tabular}{|c|c|c|c|c|c|}
\hline 05-nov-15 & Huancavelica & Lima & 2 & 3 & 30 \\
\hline 05-nov-15 & Huancayo & Huancavelica & 3 & 3 & 30 \\
\hline 05-nov-15 & Huancayo & Lima & 3 & 3 & 30 \\
\hline 05-nov-15 & Lima & Huancavelica & 3 & 3 & 40 \\
\hline 05-nov-15 & Lima & Huancayo & 3 & 3 & 30 \\
\hline
\end{tabular}

Elaboración propia

El total de viajes que se realizaron en dicho período fue de noventa y tres repartidos de la siguiente forma:

\section{Tabla 15. Resultados de total de viajes}

\begin{tabular}{|c|l|c|c|c|c|c|}
\hline & \multicolumn{5}{|c|}{ Destino } & \\
\hline \multirow{3}{*}{ Origen } & Ayacucho & Huancavelica & Huancayo & Lima \\
\hline \multirow{3}{*}{} & Ayacucho & 0 & 9 & 0 & 0 & 9 \\
\cline { 2 - 8 } & Huancavelica & 5 & 0 & 14 & 13 & 32 \\
\cline { 2 - 8 } & Huancayo & 0 & 15 & 0 & 12 & 27 \\
\cline { 2 - 8 } & Lima & 0 & 11 & 14 & 0 & 25 \\
\hline \multirow{2}{*}{ Total } & 5 & 35 & 28 & 25 & 93 \\
\hline
\end{tabular}

Elaboración propia

Figura 10. Resultados de programación

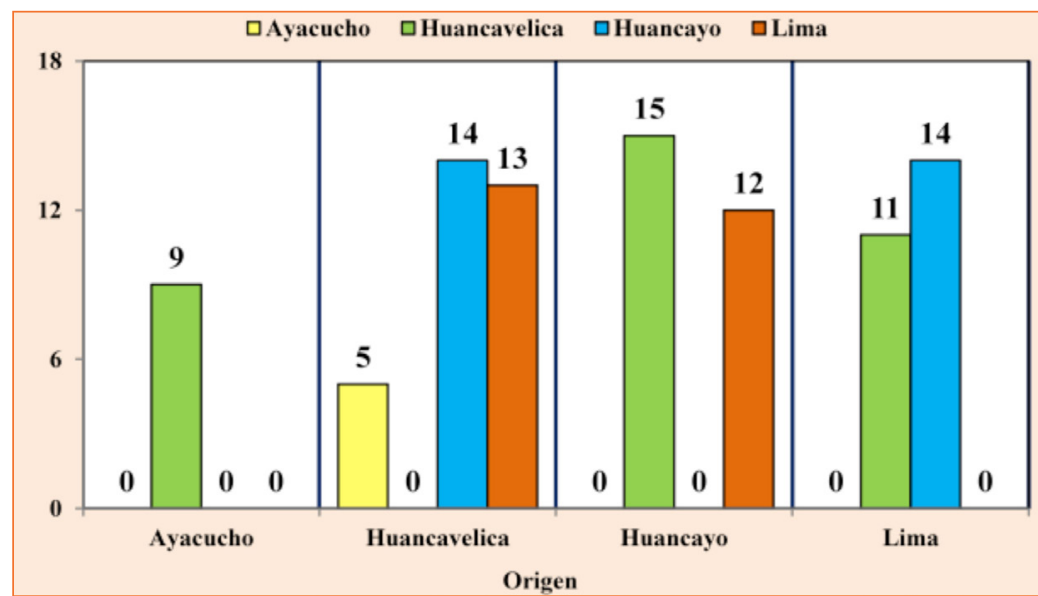

Elaboración propia 
Luego de realizar las pruebas de calidad y resultados del sistema se logra verificar que los objetivos planteados se consiguieron de la siguiente manera:

Tabla 16. Objetivos y Resultados

\begin{tabular}{|l|l|}
\multicolumn{1}{|c|}{ Objetivo } & \multicolumn{1}{c|}{ Resultado } \\
\hline $\begin{array}{l}\text { Formular el modelo matemático que permita obtener } \\
\text { una programación eficiente de los viajes con el } \\
\text { objetivo de maximizar la contribución marginal. }\end{array}$ & $\begin{array}{l}\text { Se formuló el modelo matemático como base } \\
\text { para la investigación con las respectivas } \\
\text { restricciones y el objetivo requerido. }\end{array}$ \\
\hline $\begin{array}{l}\text { Realizar pruebas para validar que los resultados del } \\
\text { modelo se ajusten a la realidad. }\end{array}$ & Se validó el modelo con un análisis de resultados. \\
\hline $\begin{array}{l}\text { Determinar el impacto de realizar una programación } \\
\text { de viajes mediante el enfoque planteado. }\end{array}$ & $\begin{array}{l}\text { Se hizo la programación de viajes mediante la } \\
\text { ejecución del modelo y el método tradicional para } \\
\text { comparar resultados. }\end{array}$ \\
\hline
\end{tabular}

Elaboración propia

\section{Observaciones, conclusiones y recomendaciones}

\subsection{Sobre los resultados}

i. El tiempo para que el modelo planteado en el software Lingo encuentre un óptimo local es de aproximadamente 15 minutos. Se puede observar que una distribución adecuada de los recursos afecta directamente en la rentabilidad de las empresas de transporte, porque al probar distintos escenarios en una programación de viajes se obtienen distintos ingresos económicos, similar conclusión a la que arribó el estudio realizado por el CIDDAT (2015).

ii. Los resultados luego del testing arrojaron una contribución marginal mayor en un 31,95 \% de la corrida del sistema frente a la programación tradicional (94 511 soles por parte del sistema y 71626 soles por parte del programador humano). Cabe señalar que los ingresos señalados son ingresos máximos esperados, siempre que se complete la capacidad de cada bus.

\subsection{Conclusiones del proyecto}

i. El sistema de soporte de decisiones para una empresa de transporte terrestre interprovincial, desarrollado en la investigación, sirvió para mejorar el proceso de programación de viajes y así tomar mejores decisiones al momento de hacer sus planificaciones. 
ii. Una adecuada programación de viajes en este tipo de empresas mejora significativamente el manejo de sus recursos, la toma de decisiones y la rentabilidad.

iii. La aplicación de revenue management en el proyecto estuvo enfocada en la fijación de precios para alinear la demanda estimada a la oferta de la empresa, y así obtener una mejor contribución marginal.

\subsection{Recomendaciones}

Se recomienda la implementación de un sistema para pronosticar la demanda y mejorar la calidad de los resultados.

\section{Referencias}

Cross, R. (1997). Revenue Management: Hard-Core Tactics for Market Domination. Crown Business. Nueva York: Broadway Books.

Eom, S. y Kim, E. (2006). A Survey of Decision Support System Applications (1995-2001). The Journal of the Operational Research Society, 57(11), 1264-1278. DOl:10.1057/palgrave. jors. 2602140

Forgacs, G. (2010). Revenue Management: Maximizing Revenue in Hospitality Operations. Michigan: American Hotel \& Lodging Educational Institute.

Gallego, G., Ryan, J., y Simchi-Levi, D. (2001). Minimax Analysis for Finite-Horizon Inventory Models. IIE Transactions, 33(10), 861-874. DOI: 10.1023/A: 1010974605771

Jaramillo, M. (2012). Network Revenue Management en aerolíneas resuelto a través de programación dinámica robusta (tesis doctoral). Universidad de Chile, Santiago de Chile, Chile. Recuperado de http://repositorio.uchile.cl/bitstream/ handle/2250/112043/cfjaramillo_mq.pdf?sequence=1\&isAllowed=y

Karadjov, Y., y Farahmand, M. (2007). Revenue Management Circa 2020. Journal of Revenue and Pricing Management, 6(4), 291-292. DOl: 10.1057/ palgrave.rpm.5160100

Keen, P. y Scott, M. (1978). Decision Support Systems: an Organizational Perspective. Massachusets: Addison-Wesley Pub.

Mainzer, B. (2004). Future of Revenue Management. Fast Forward for Hospitality Revenue Management. Journal of Revenue and Pricing Management, 3(3), 285-289.

Merino, M. (2015). Técnicas clásicas de optimización. Parte l: programación lineal y no lineal. Recuperado de http://www.ehu.eus/mae/html/prof/Maria_archivos/ plnlapuntes.pdf 
Prats, L., y Guia, J. (2012). Gestión de precios en un sistema de Revenue Management hotelero en línea. Pasos, 10(5), 511-520.

Ruiz, Hernández, L., Giraldo, W. (2009). Aplicación de los sistemas de soporte a la decisión (DSS) en el comercio electrónico. Revista Ingeniería e Investigación, 29 (2), 94-99. Recuperado de: http://www.scielo.org.co/pdf/iei/v29n2/v29n2a15.pdf.

Romainville, M. (2015, 11 de diciembre). Las 9 empresas de transporte terrestre más grandes del Perú. El Comercio. Recuperado de http://elcomercio.pe/economia/peru/ 9-empresastransporte-terrestre-mas-grandes-peru-noticia-1863039/

Ryzin, G. y McGill, J. (2000). Revenue Management without Forecasting or Optimization: an Adaptive Algorithm for Determining Airline Seat Protection Levels. Management Science, 46(6), 760-775.

Steed, E., y Gu, Z. (2005). An Examination of Hotel Room Pricing Methods: Practised and Proposed. Joumal of Revenue and Pricing Management, 3(4), 369-379. DOl: 10.1057/palgrave.rpm.5170121

Turban, E. (1995). Decision Support and Expert Systems: Management Support Systems (4a ed.). Nueva Jersey: Prentice Hall.

Turban, E., Aronson, J., y Liang, T. (2005). Decision Support Systems and Intelligent Systems. Nueva Jersey: Prentice Hall.

Vinod, B. (2004). Unlocking the Value of Revenue Management in the Hotel Industry. Journal of Revenue and Pricing Management, 3(2),178-190. DOl: 10.1057/ palgrave.rpm.5170105

Weatherford, L., y Belobaba, P. (2002). Revenue Impacts of Fare Input and Demand Forecast Accuracy in Airline Yield Management. The Journal of the Operational Research Society, 53(8), 811-821. DOl: 10.1057/palgrave.jors.2601357

Yeoman, I. (2001). Yield Management: Strategies for the Service Industries. En Ingold, A., y Mc Mahon, U. (eds). Londres: Cengage Learning.

\section{Glosario}

Lingo: Software diseñado para la construcción de soluciones lineales, no lineales y enteras alcanzando la optimización de forma rápida.

Revenue management: Aplicación de herramientas de análisis que ayudan a reconocer comportamientos e identificar tendencias de usuarios o clientes por medio del manejo del precio y la demanda.

Sistema de soporte de decisiones: Sistema informático de apoyo para la toma de decisiones.

Testing: Proceso de evaluación. 